\title{
The possibility of ideological bias in structural macroeconomic models
}

\author{
Gilles Saint-Paul \\ Toulouse school of economics
}

June 8, 2011

\section{Introduction}

Can ideological bias pervade economic modelling, and yet act in such a way that prevailing models remain consistent with the data? Such biases may explain ongoing controversies among macroeconomists about key structural parameters, in particular (i) the size of the Keynesian multiplier, (ii) the slope of the aggregate supply curve, and (iii) the nature of the shocks that drive business cycles.

These controversies are well known to our profession. Here is an excerpt from a web reading list (http://homepage3.nifty.com/ronten/crisisreadings.htm) about the financial crisis:

The Spending Multiplier Debate

Positive: Romer and Bernstein: The Job Impact of the American Recovery and Reinvestment Plan; Krugman: The Conscience of a Liberal; Getting fiscal; War and Non-Remembrance; Don't know much about history; Paul Krugman recommends us to learn more from Japan's experiences; Adam P. Posen (1998) Fiscal 
Policy Works When It Is Tried in Restoring Japan's Economic Growth.

Skeptical: Mankiw: Fiscal Policy Puzzles; *Spending and Tax Multipliers, How Not to Stimulate the Economy; John Taylor: Why Permanent Tax Cuts Are the Best Stimulus; Cogan, Cwik, Taylor and Wieland (2009): New Keynesian versus Old Keynesian Government Spending Multipliers; Smets-Wouters (2003) Model; Hall and Woodford: Measuring the Effect of Infrastructure Spending on GDP with a comment by Robert Gordon.

Now here are some excerpts from the wikipedia articles about some of the above authors:

Romer: "Christina Romer (née Duckworth; born December 25, 1958) is the Class of 1957 Garff B. Wilson Professor of Economics at the University of California, Berkeley and the out-going Chair of the Council of Economic Advisers in the Obama administration. She resigned from her role on the Council of Economic Advisers on September 3, 2010. After her nomination and before the Obama administration took office, Romer worked with economist Jared Bernstein to co-author the administration's plan recovery from the 2008 recession. In a January 2009 video presentation, she discussed details of the job creation package that the Obama administration submitted to Congress."

Krugman: "In a review for The New York Times, Pulitzer prize-winning historian David M. Kennedy stated, "Like the rants of Rush Limbaugh or the films of Michael Moore, Krugman's shrill polemic may hearten the faithful, but it will do little to persuade the unconvinced" 
Mankiw: "He returned to politics when he was appointed by President George W. Bush as Chairman of the Council of Economic Advisors in May 2003."

Taylor: "He has been active in public policy, serving as the Under Secretary of the Treasury for International Affairs during the first term of the George W. Bush Administration"

These quotes suggest a congruence between political preferences and views about economic parameters. ${ }^{1}$ It is also true, historically, that left-wing economists have leaned toward a "flat" Phillips curve, while conservatives prefer it steep or even vertical. In the eighties, when the RBC literature was being developed, "fresh water" conservatives emphasized the quantitative role of demand shocks, while "salt water" social democrats thought that demand shocks were the driving force of economic fluctuations.

This paper studies the trade-offs that an expert with ideological biases faces in designing his model. As in Saint-Paul (2011), I assume the perceived model must be autocoherent, in that its use by all agents delivers a selfconfirming equilibrium (as in Fudenberg and Levine $(2003,2007)$ and Sargent (2008)). The exercise is carried in the context of a simplified AS-AD model, where in principle the expert can influence policy by manipulating six key parameters:

-The response of aggregate demand to government expenditure

-The response of aggregate demand to interest rates

-The response of output to actual inflation in the Phillips curve

-The response of output to expected inflation

-The variance of supply shocks

-The variance of demand shocks

Do we expect the economists's political preferences to influence those

\footnotetext{
${ }^{1}$ To some extent, Fuchs et al. (1999) report similar findings.
} 
parameters in the directions predicted by the above discussion? The answer is a rough yes. For example, a larger reported Keynesian multiplier is favored by more left-wing economists, because it induces the government to pursue more activist policies, which they like better than conservatives. Similarly, a flatter inflation output trade-off will increase the perceived efficiency of activist policies, and left-wing economists will also want to favor those configurations.

But an important aspect of the analysis is that autocoherence conditions imply constraints and trade-offs between parameters. For example a larger reported Keynesian multiplier must be associated with a lower interest elasticity of aggregate demand for the economists's model to match the data, otherwise the covariance between output and an observed leading indicator of activity will be missed. Consequently, being over-optimistic about fiscal policy implies being over-pessimistic about monetary policy. Similarly, the economist must often run against his preferences for the short-term Phillips curve or the relative variance of supply shocks for the autocoherence constraints to be met.

Furthermore, some parameters or some combinations of parameters must be truthfully revealed for the expert to remain autocoherent. These are the parameters that are "identified" from the empirical moments of the distribution of observables that the economist must match. In the simple example below, agents base their expectations on a signal of the underlying demand shock, and that signal is orthogonal to the supply disturbance in the Phillips curve. This allows private agents to implicitly estimate the correct long-run slope of the Phillips curve by using that signal as an instrument; in other words, the long-run slope of the Phillips curve is implicitly revealed by the equilibrium moments of the observables. As it turns out, only that long-run slope matters for policy, and it is then impossible for economists to influence policy through the perceived Phillips curve parameters. I then extend the model to allow for this possibility, by assuming that the agents' signal is pol- 
luted by the supply shock. This illustrates the tight link between parameter identification and the scope for bias that is generated by the autocoherence conditions.

Another insight delivered by the analysis below is the possibility of what I label as criticality. For some parameter values of the true model, the autocoherence conditions imposed on the perceived model may make it locally impossible for the expert to influence policy. In such a case even small deviations between the expert's ideological preferences and the government will lead to large differences in the reported parameter values.

\section{An AS-AD model}

The economy is driven by a standard AS-AD structure. Two kinds of agents make decisions: the government and the people. The government sets government expenditures, while the people's decisions depend on their inflationary expectations. Both use a perceived model which will be determined by a single self-serving economist. Therefore, we will distinguish between the correct model $(\mathrm{CM})$, and the perceived one $(\mathrm{PM})$, whose parameters are denoted with a hat.

The model consists of three equations:

$$
\begin{aligned}
y & =-\beta i+\alpha g+u_{0}+\theta v \\
i & =p+y \\
y & =\delta p-\mu p^{e}+v
\end{aligned}
$$

The endogenous variables are $y$, output, $g$, public expenditure, $i$, the interest rate, $p$, the price level, and $p^{e}$, the expected price level (to make the discussion more realistic I will interchangeably refer to $p$ as the inflation rate). Therefore the model is closed if a rule for forming expectations and a policy rule are added to these three structural equations. 
The economy is subjected to an aggregate demand shock $u_{0}$ and an aggregate supply shock $v$. The first equation is an "IS" curve, the second one can be interpreted as either an LM curve or a Taylor rule ${ }^{2}$, and the third equation is an aggregate supply (or Phillips) curve. Note that the supply shock also affects aggregate demand. This makes it harder to identify the true model's parameters and raises the expert's degrees of freedom in designing his model. There is no dearth of theoretical mechanisms for supply shocks to affect aggregate demand as well; in most models greater productivity will change investment and consumption plans through its relative price and wealth effects. The coefficients of the interest rate equation are assumed to be common knowledge and normalized to one for simplicity.

I assume $0 \leq \mu \leq \delta$. Roughly, $\delta$ can be interpreted as the slope of the short-run Phillips curve and $\delta-\mu$ as the slope of the long-run Phillips curve. If $\delta=\mu$, we have a Lucas supply curve, and there is no long-run trade-off between output and inflation. If $\mu=0$, we have an old fashioned Phillips curve which ignores expectations. The output-inflation trade-off is more "favorable", the greater $\delta$ and the smaller $\mu$. Intuitively, we might expect more "progressive" experts to favor models with large values of $\delta$ and small values of $\mu$. The other parameters of interest are $\alpha$, referred to as the "demand Keynesian multiplier" (DKM), and $\beta$, the interest elasticity of aggregate demand. These two parameters are nonnegative.

Eliminating interest rates, the model can be re-expressed as the following recursive form:

$$
\begin{aligned}
y & =-b p^{e}+a g+u+\rho v \\
p & =\frac{\mu}{\delta} p^{e}-\frac{v}{\delta}+\frac{y}{\delta}
\end{aligned}
$$

\footnotetext{
${ }^{2}$ In this static model, we do not have to worry about the actual values of the coefficients of the interest rate rule. Here they are normalized tio one to save on notation.
} 
Here, $a, b$, and $\rho$ are composite parameters given by

$$
\begin{aligned}
a & =\frac{\alpha \delta}{\delta+\beta(1+\delta)} \\
b & =\frac{\beta \mu}{\delta+\beta(1+\delta)} \leq \mu \\
\rho & =\frac{\beta+\theta \delta}{\delta+\beta(1+\delta)} \geq \frac{b}{\mu} .
\end{aligned}
$$

To save on notation, the aggregate demand shock is redefined as $u=$ $\frac{\delta}{\delta+\beta(1+\delta)} u_{0}$.

Both expectations and government policy are formed upon observing a signal of the demand shock,

$$
z=\omega u+\varepsilon
$$

where $\varepsilon$ is noise. I assume

$$
(u, v, \varepsilon) \sim N\left(0,\left(\begin{array}{ccc}
\sigma_{u}^{2} & 0 & 0 \\
0 & \sigma_{v}^{2} & 0 \\
0 & 0 & \sigma_{\varepsilon}^{2}
\end{array}\right)\right) .
$$

Furthermore, to simplify on notation I will impose the normalization

$$
\omega^{2} \sigma_{u}^{2}+\sigma_{\varepsilon}^{2}=1
$$

After the equilibrium is realized, people observe the output level $y$ and the price level $p$. Given that the monetary policy rule is known and the interest rate only depends on $p$ and $y$, there is no additional information in observing the interest rate.

Thus, we distinguish between two information sets: The information set prevailing when expectations and government policy are formed, which is given by $\{z\}$, and the information set which determines the data against which any credible model must be validated. That information set is given 
by $\{y, p, z\} .{ }^{3}$ Government spending is also observed but since it will be proportional to $z$, with a slope parameter which is common knowledge, that knowledge is redundant.

The perceived model must satisfy the plausibility conditions that all its parameters are nonnegative and that $0 \leq \hat{\mu} \leq \hat{\delta}$. Since, given the other parameters, any plausible target value for $(\hat{a}, \hat{b}, \hat{\rho})$ that satisfies $\hat{\rho} \geq \frac{\hat{b}}{\hat{\mu}}$ and $\hat{b} \leq \hat{\mu}$ can be matched by an appropriate choice of $(\hat{\alpha}, \hat{\beta}, \hat{\theta})$, I will consider that the theorist can directly set the three composite parameters $(\hat{a}, \hat{b}, \hat{\rho})$, and accordingly add the inequalities $\hat{\rho} \geq \frac{\hat{b}}{\hat{\mu}}$ and $\hat{b} \leq \hat{\mu}$ to the set of plausibility conditions.

I will proceed as follows. First, I solve for the equilibrium, given the model used by the people and the level of government spending. Second, I derive the optimal government policy. Third, I spell out the autocoherence conditions that the perceived model must satisfy.

\section{Solution}

\subsection{Solving for $p$ and $y$.}

The first step in solving for the equilibrium consists in computing $p^{e}$. Substituting (1) into (2) we get that

$$
p=\frac{\mu}{\delta} p^{e}-\frac{v}{\delta}-\frac{b}{\delta} p^{e}+\frac{a}{\delta} g+\frac{u}{\delta}+\frac{\rho v}{\delta} .
$$

People believe that the following relationship holds instead:

$$
p=\frac{\hat{\mu}}{\hat{\delta}} p^{e}-\frac{\hat{v}}{\hat{\delta}}-\frac{\hat{b}}{\hat{\delta}} p^{e}+\frac{\hat{a}}{\hat{\delta}} g+\frac{\hat{u}}{\hat{\delta}}+\frac{\hat{\rho} \hat{v}}{\hat{\delta}}
$$

\footnotetext{
${ }^{3}$ Note that I require the model to match those data despite that it will be used prior to their realization. While the model is one-shot, I want it to take into account the fact that the people's forecasting model will be used repeatedly and therefore must match the data.
} 
Note the hats on $u$ and $v$ : the realization of the shocks that would be inferred from the people's model differ from the actual ones, unless the model is correct.

To obtain $p^{e}$ we take expectations on both sides, using the conditional distributions generated by the perceived model. I denote again by a hat this expectation. We get that

$$
p^{e}=\frac{1}{\hat{\delta}+\hat{b}-\hat{\mu}} \hat{E}(u \mid z)+\frac{\hat{a}}{\hat{\delta}+\hat{b}-\hat{\mu}} g .
$$

Substituting into (1), we get a reduced form equation for output

$$
y=-\frac{b}{\hat{\delta}+\hat{b}-\hat{\mu}} \hat{E}(u \mid z)+\left(a-\frac{b \hat{a}}{\hat{\delta}+\hat{b}-\hat{\mu}}\right) g+u+\rho v .
$$

Plugging (3) and (4) into (2), we then get

$$
p=\frac{\mu-b}{\delta(\hat{\delta}+\hat{b}-\hat{\mu})} \hat{E}(u \mid z)+\left(\frac{a}{\delta}+\frac{\hat{a}(\mu-b)}{\delta(\hat{\delta}+\hat{b}-\hat{\mu})}\right) g+\frac{u}{\delta}+\frac{\rho-1}{\delta} v .
$$

\subsection{Optimal government policy}

As in Saint-Paul (2011), the government wants to stabilize output and government spending. Its objective function is

$$
\min \hat{E}\left(y^{2}+\varphi g^{2}\right),
$$

where $\varphi$ is a parameter which captures how conservative the government is. I could also allow for the government to stabilize prices, but since the government can only react to demand shocks - there is no supply signal at the time of setting policy - that additional objective is similar to stabilizing output, and I ignore it for simplicity. ${ }^{4}$

\footnotetext{
${ }^{4}$ One could extend the model by assuming that a signal of the supply shock is also observed. Responding to that signal would involve a trade-off between price stability and output stability. In this paper the focus is instead on price/output stability vs. government expenditure stability.
} 
Upon realization of the signal $z$, the government sets $g$ so as to minimize

$$
\hat{E}\left(y^{2}+\varphi g^{2} \mid z\right)=\hat{E}\left(y^{2} \mid z\right)+\varphi g^{2} .
$$

I assume $g$ is observed at the time of setting inflationary expectations. Therefore, there is no credibility problem and the government will internalize the entire feedback effect of fiscal stimulus on output through inflation and its monetary policy response when setting its policy ${ }^{5}$. Therefore, the first-order condition is

$$
\frac{\hat{d} y}{\hat{d} g} \hat{E}(y \mid z)+\varphi g=0 .
$$

The derivative $\frac{\hat{d} y}{\hat{d} g}$ is the perceived reduced form Keynesian multiplier (RFKM), which is different from the impact multiplier (IKM), itself equal to $a$ and perceived as $\hat{a}$. Its correct value can be obtained from (4):

$$
\frac{d y}{d g}=a-\frac{b \hat{a}}{\hat{\delta}+\hat{b}-\hat{\mu}} .
$$

Two aspects are noteworthy. First, the true Keynesian multiplier not only depends on the true model but also on the perceived one. This is because part of the expansionary effect of government spending is dissipated by greater inflationary expectations, which in turn generate greater inflation and a contractionary response of the interest rate. For example, the more people believe that government policy is effective (the greater $\hat{a}$ ), the more they think it will be inflationary, and the smaller the Keynesian multiplier given $a$. For the same reason, the more people believe the output/inflation trade-off is unfavorable (the smaller $\hat{\delta}$ ), the smaller $\frac{d y}{d g}$. Second, the Keynesian multiplier is not identified, because $g$ is endogenous and always proportional

\footnotetext{
${ }^{5}$ In Saint-Paul (2011), I discuss how parameter manipulation can be a way for a "benevolent" economist to provide the government with a commitment device.
} 
to $z$. If there was a random, exogenous component to $g$, and if that component were observable, it would make it possible to identify the Keynesian multiplier. That is, the vector space spanned by $g$ and $z$ would be of dimension 2 instead of 1 . Here, though, people cannot disentangle the sensitivity of output to government spending from the direct effect of demand shocks. Similar considerations arise in Sargent (2008) and Fudenberg and Levine (2003, 2007). This underidentification would still hold in richer models provided that the number of parameters is large enough relative to the dimension of the observables space.

The government uses the perceived model to compute the Keynesian multiplier. To get the perceived multiplier, one just has to replace $a$ and $b$ with $\hat{a}$ and $\hat{b}$, respectively, in (7), getting

$$
\frac{\hat{d} y}{\hat{d} g}=\frac{\hat{a}(\hat{\delta}-\hat{\mu})}{\hat{\delta}+\hat{b}-\hat{\mu}} .
$$

To compute $g$, we can compute $\hat{E}(y \mid z)$ by applying hatted expectations to (4), yielding

$$
\hat{E}(y \mid z)=\frac{\hat{a}(\hat{\delta}-\hat{\mu})}{\hat{\delta}+\hat{b}-\hat{\mu}} g+\frac{\hat{\delta}-\hat{\mu}}{\hat{\delta}+\hat{b}-\hat{\mu}} \hat{E}(u \mid z) .
$$

By Bayes' law, we have

$$
\hat{E}(u \mid z)=\frac{\hat{\omega} \hat{\sigma}_{u}^{2}}{\hat{\omega} \hat{\sigma}_{u}^{2}+\hat{\sigma}_{\varepsilon}^{2}} z .
$$

As will be shown below, autocoherence implies that

$$
\begin{aligned}
1 & =E z^{2}=\hat{E} z^{2} \\
& =\omega^{2} \sigma_{u}^{2}+\sigma_{\varepsilon}^{2} \\
& =\hat{\omega}^{2} \hat{\sigma}_{u}^{2}+\hat{\sigma}_{\varepsilon}^{2} .
\end{aligned}
$$

For simplicity I will make use of this right away. Then 


$$
\hat{E}(u \mid z)=\hat{\omega} \hat{\sigma}_{u}^{2} z .
$$

Substituting (10),(9), and (8) into (6), we eventually get

$$
g=\gamma z
$$

where

$$
\gamma=-\hat{a} \frac{(\hat{\delta}-\hat{\mu})^{2}}{\varphi(\hat{\delta}+\hat{b}-\hat{\mu})^{2}+\hat{a}^{2}(\hat{\delta}-\hat{\mu})^{2}} \hat{\omega} \hat{\sigma}_{u}^{2}<0 .
$$

Inspection of this formula reveals that government activism is larger, i.e. $|\gamma|$ is larger,

- The more people believe in a favorable "long-term" Phillips curve, i.e. the greater $\hat{\delta}-\hat{\mu}$

- The more they believe the interest response of aggregate demand is low, i.e. the smaller $\hat{b}$

As for the effect of the perceived IKM $\hat{a}$, there is an "income effect" and a"substitution" effect, implying that $\gamma$ is not monotonic in $\hat{a}$. For small values of $\hat{a}$, the substitution effect dominates; a more efficient fiscal policy generates greater activism. For large values of $\hat{a}$, though, the income effect dominates: the government takes advantage of an increase in $\hat{a}$ to reduce its activism, since that increase has a direct favorable impact of the degree of stabilization which is being achieved.

\subsection{The reduced form model}

The preceding subsection allows to compute the variables of interest $p$ and $y$ as a function of the realization of the shocks $u, v$ and $\varepsilon$. This solution determines the reduced form model, which is summarized in Table 1. Then, 
by replacing non hatted parameters (other than $\gamma$ ) by their hatted counterparts, one can compute the reduced form perceived model, which is reported in Table 2. These expressions introduce composite coefficients that capture the response of output and prices to the demand shock $u$ and the error $\varepsilon$.

For example, the coefficient of $u$ on $y$,

$$
a_{y u}=-\frac{b}{\hat{\delta}+\hat{b}-\hat{\mu}} \omega \hat{\omega} \hat{\sigma}_{u}^{2}+\omega \gamma\left(a-\frac{\hat{a} b}{\hat{\delta}+\hat{b}-\hat{\mu}}\right)+1,
$$

has three components. The constant 1 captures the direct effect of the aggregate demand shock on output. The term $\omega \gamma\left(a-\frac{\hat{a} b}{\hat{\delta}+\hat{b}-\hat{\mu}}\right)$ is typically negative and captures the stabilizing effect of fiscal policy. In it, $a$ captures the direct effect of fiscal policy, and $-\frac{\hat{a} b}{\hat{\delta}+\hat{b}-\hat{\mu}}$ captures the dissipation due to the effect of fiscal policy on price expectations: A fiscal expansion boosts expectations of inflation, which in turn increases actual inflation and interest rates, which in turn reduces the efficiency of the expansion ${ }^{6}$. This reaction is stronger, the greater the perceived effect of fiscal policy on output $(\hat{a})$, the greater the actual effect of interest rates on output (b), and the more "unfavorable" the perceived Phillips curve (the greater $\hat{\mu}$ and the smaller $\hat{\delta}$ ). Finally, the term $-\frac{b}{\delta+\hat{b}-\hat{\mu}} \omega \hat{\omega} \hat{\sigma}_{u}^{2}$ is the effect of the direct reaction of price expectations to the signal about the demand shock. Since this effect would not exist absent a reaction of interest rates to inflation, we will label it the "monetary component" of the reaction of output to demand shocks. It is also negative, since that effect tends to dampen demand shocks.

\footnotetext{
${ }^{6}$ If people think that fiscal policy is much more efficient than it actually is, i.e. if $\hat{a}$ is large, then the fiscal term becomes positive, implying that fiscal expansions are contractionary because of their (excessive) adverse effect on inflation expectations.
} 


\begin{tabular}{ll}
\hline \hline Observable & Expression \\
\hline Output & $y=a_{y u} u+a_{y \varepsilon} \varepsilon+\rho v$ \\
Price & $p=a_{p u} u+a_{p \varepsilon} \varepsilon+\frac{\rho-1}{\delta} v$ \\
\hline \hline Coefficients & Expression \\
\hline$a_{y u}$ & $-\frac{b}{\hat{\delta}+\hat{b}-\hat{\mu}} \omega \hat{\omega} \hat{\sigma}_{u}^{2}+\omega \gamma\left(a-\frac{\hat{a} b}{\hat{\delta}+\hat{b}-\hat{\mu}}\right)+1$ \\
$a_{y \varepsilon}$ & $-\frac{b}{\hat{\delta}+\hat{b}-\hat{\mu}} \hat{\omega} \hat{\sigma}_{u}^{2}+\gamma\left(a-\frac{\hat{a} b}{\hat{\delta}+\hat{b}-\hat{\mu}}\right)$ \\
$a_{p u}$ & $\frac{\mu-b}{\delta(\hat{\delta}+\hat{b}-\hat{\mu})} \omega \hat{\omega} \hat{\sigma}_{u}^{2}+\omega \gamma\left(\frac{a}{\delta}+\frac{\hat{a}(\mu-b)}{\delta(\hat{\delta}+\hat{b}-\hat{\mu})}\right)+\frac{1}{\delta}$ \\
$a_{p \varepsilon}$ & $\frac{\mu-b}{\delta(\hat{\delta}+\hat{b}-\hat{\mu})} \hat{\omega} \hat{\sigma}_{u}^{2}+\left(\frac{a}{\delta}+\frac{\hat{a}(\mu-b)}{\delta(\hat{\delta}+\hat{b}-\hat{\mu})}\right) \gamma$ \\
\hline \hline
\end{tabular}

Table 1 - The correct reduced form model

\begin{tabular}{|c|c|}
\hline Observable & Expression \\
\hline Output & $y=\hat{a}_{y u} \hat{u}+\hat{a}_{y \varepsilon} \hat{\varepsilon}+\hat{\rho} \hat{v}$ \\
\hline Price & $p=\hat{a}_{p u} \hat{u}+\hat{a}_{p \varepsilon} \hat{\varepsilon}+\frac{\hat{\rho}-1}{\hat{\delta}} v$ \\
\hline Coefficients & Expression \\
\hline$\hat{a}_{y u}$ & $-\frac{\hat{b}}{\hat{\delta}+\hat{b}-\hat{\mu}} \hat{\omega}^{2} \hat{\sigma}_{u}^{2}+\gamma \hat{\hat{\omega}} \frac{\hat{a}(\hat{\delta}-\hat{\mu})}{\hat{\delta}+\hat{b}-\hat{\mu}}+1$ \\
\hline$\hat{a}_{y \varepsilon}$ & $-\frac{\hat{b}}{\hat{\delta}+\hat{b}-\hat{\mu}} \hat{\omega} \hat{\sigma}_{u}^{2}+\gamma \frac{\hat{a}(\hat{\delta}-\hat{\mu})}{\hat{\delta}+\hat{b}-\hat{\mu}}$ \\
\hline$\hat{a}_{p u}$ & $\frac{\hat{\mu}-\hat{b}}{\hat{\delta}(\hat{\delta}+\hat{b}-\hat{\mu})} \hat{\omega}^{2} \hat{\sigma}_{u}^{2}+\frac{\hat{a}}{\hat{\delta}+\hat{b}-\hat{\mu}} \hat{\omega} \gamma+\frac{1}{\hat{\delta}}$ \\
\hline$\hat{a}_{p \varepsilon}$ & $\frac{\hat{\mu}-\hat{b}}{\hat{\delta} \hat{\delta}+\hat{b}-\hat{\mu})} \hat{\omega} \hat{\sigma}_{u}^{2}+\frac{\hat{a}}{\hat{\delta}+\hat{b}-\hat{\mu}} \gamma$ \\
\hline
\end{tabular}

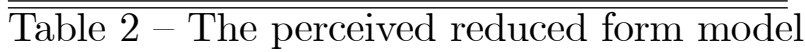

\section{Autocoherence conditions}

The reduced form models can then be used to derive the autocoherence conditions. The perceived model must correctly predict the joint distribution of the observables. As all variables are Gaussian and it is common knowledge that their mean is zero, the autocoherence property requires that the variance-covariance matrix of $(y, p, z)^{\prime}$ computed using that perceived model matches the actual one. This determines six independent autocoherence conditions that are derived in the Appendix (equations (22)-(27)). There are nine parameters: $\left(\hat{a}, \hat{b}, \hat{\rho}, \hat{\sigma}_{u}^{2}, \hat{\delta}, \hat{\mu}, \hat{\sigma}_{v}^{2}, \hat{\omega}, \hat{\sigma}_{\varepsilon}^{2}\right)$ and therefore three degrees of 
freedom.

Since the joint distribution of $p$ and $z$ is observed, the autocoherence conditions always imply that $\hat{E}(p \mid z)=E(p \mid z)$. In other words, in equilibrium expectations are rational in the usual sense ${ }^{7}$. If government policy were fixed, we could then solve for a unique rational expectations equilibrium (REE) for model (1)-(2) in the usual way. All autocoherent models would then be equivalent in that they deliver the same REE equilibrium ${ }^{8}$, leaving no room for the economists to manipulate outcomes. However, government policy does depend on the perceived model, because to set its optimal policy the government must know structural parameters (in particular the multiplier $a)$ that are not identified from the joint distribution of $(p, y, z)$. This opens the possibility for the expert to manipulate government policy.

However, not all parameters can be used to manipulate policy. The autocoherence conditions imply that the parameters of the Phillips curve are useless for pursuing an agenda.

Proposition 1 — The autocoherence conditions imply

$$
\hat{\delta}-\hat{\mu}=\delta-\mu
$$

Proof - See Appendix.

\footnotetext{
${ }^{7}$ Algebraically, we have that $E(p \mid z)=a_{p u} E(u \mid z)+a_{p \varepsilon} E(u \mid z)$

$=\left(a_{p u} \omega \sigma_{u}^{2}+a_{p \varepsilon} \sigma_{\varepsilon}^{2}\right) z$. Similarly, $\hat{E}(p \mid z)=\left(\hat{a}_{p u} \hat{\omega} \hat{\sigma}_{u}^{2}+\hat{a}_{p \varepsilon} \hat{\sigma}_{\varepsilon}^{2}\right) z$. Therefore, the condition $E(u \mid z)=\hat{E}(u \mid z)$ is equivalent to $a_{p u} \omega \sigma_{u}^{2}+a_{p \varepsilon} \sigma_{\varepsilon}^{2}=\hat{a}_{p u} \hat{\omega} \hat{\sigma}_{u}^{2}+\hat{a}_{p \varepsilon} \hat{\sigma}_{\varepsilon}^{2}$, i.e. autocoherence condition (AC3) in the appendix.

${ }^{8}$ Again, this can be checked algebraically. Note that $\frac{\omega \sigma_{u}^{2}}{\delta}+a_{p \varepsilon}=\frac{\omega \sigma_{u}^{2}}{\delta}+\frac{\gamma a}{\delta}+$ $\frac{\mu-b}{\delta}\left[\frac{\hat{\omega} \hat{\sigma}_{u}^{2}+\gamma \hat{a}}{\hat{\delta}+\hat{b}-\hat{\mu}}\right]$ and that $\frac{\hat{\omega} \hat{\sigma}_{u}^{2}}{\hat{\delta}}+\hat{a}_{p \varepsilon}=\frac{\hat{\omega} \hat{\sigma}_{u}^{2}+\gamma \hat{a}}{\hat{\delta}+\hat{b}-\hat{\mu}}$. Therefore, condition (24) is equivalent to $\frac{\hat{\omega} \hat{\sigma}_{u}^{2}+\gamma \hat{a}}{\hat{\delta}+\hat{b}-\hat{\mu}}=\frac{\omega \sigma_{u}^{2}+\gamma a}{\delta+b-\mu}$. Next, note that all the hatted terms in $a_{p \varepsilon}, a_{p u}, a_{y \varepsilon}$ and $a_{y u}$ can be grouped in the ratio $\frac{\hat{\omega} \hat{\sigma}_{u}^{2}+\gamma \hat{a}}{\hat{\delta}+\hat{b}-\hat{\mu}}$. Since that ratio must be equal to $\frac{\omega \sigma_{u}^{2}+\gamma a}{\delta+b-\mu}$, if $\gamma$ is exogenous, none of those coefficients depend on the perceived model. Consequently, the equilibrium is unique and must be identical to the REE equilibrium.
} 
Corollary - Given $\hat{a}$, and $\hat{b}, \gamma$ is independent of the choice of $\hat{\delta}$ and $\hat{\mu}$, and so is the equilibrium.

Proof - Immediate from (11).

The policymaker cares about the ultimate effect of output of government spending, which only depends on price formation through the difference between the output response to prices $\delta$ and its (adverse) response to price expectations $\mu$. But to match the covariances between output and the demand signal and prices and the demand signal, the economist is forced to reveal this difference. Thus given $\hat{a}$ and $\hat{b}$, he cannot influence policy through the design of the price block of his model. ${ }^{9}$ Intuitively, this is because the demand signal $z$, which is not polluted by the supply shock, acts as an instrumental variable allowing agents to infer $\delta-\mu$ from $\operatorname{cov}(y, z)$ and $\operatorname{cov}(p, z)$, two empirical moments that must be correctly predicted by the perceived model.

Since there is little room for the perceived Phillips curve to be used by expert to influence outcomes, in what follows I will assume that $\delta$ and therefore $\mu$ are known. In the subsequent section I will discuss a case where Proposition 1 does not hold and policy can be influenced through the perceived Phillips curve parameters.

\section{The price block is revealed}

In this section I assume $\delta$ and $\mu$ are known. Furthermore, to simplify the analysis, I will also assume that $\omega$ and $\sigma_{u}$ are known. Note that the only way for the expert to affect the perceived values of $a$ and $b$ is then through the

\footnotetext{
${ }^{9}$ Remember, though, that $\hat{a}$ and $\hat{b}$ are themselves composite parameters and their expression depends on $\hat{\delta}$ and $\hat{\mu}$. While given $\hat{\delta}$ and $\hat{\mu}$, any target for those parameters can be reached by picking the appropriate $\hat{\alpha}$ and $\hat{\beta}$, if for example $\alpha$ is known it may be necessary to choose a particular value of $\hat{\delta}$ to get the desired value of $\hat{a}$.
} 
perceived underlying demand Keynesian multiplier and interest elasticity of aggregate demand. For the sake of simplicity, in the following discussion I will assimilate a change in $\hat{a}$ with a change in $\hat{\alpha}$ in the same direction, and similarly for $\hat{b}$ and $\hat{\beta}$.

\subsection{Simplifying the autocoherence conditions}

Under our assumptions, it must be that $\hat{\omega}=\omega, \hat{\sigma}_{u}=\sigma_{u}, \hat{\delta}=\delta$, and $\mu=\hat{\mu}$. It is then shown in the Appendix that in such a case, autocoherence implies that the perceived reduced form model must match the correct reduced form model, that is:

$$
\begin{aligned}
a_{y \varepsilon} & =\hat{a}_{y \varepsilon}, \\
a_{y u} & =\hat{a}_{y u}, \\
a_{p \varepsilon} & =\hat{a}_{p \varepsilon}, \\
a_{p u} & =\hat{a}_{p u}, \\
\rho & =\hat{\rho} .
\end{aligned}
$$

Nevertheless, because the correct reduced form coefficients themselves depend on beliefs, through the government policy parameter $\gamma$, it does not follow that the perceived structural model should be the same as the correct one. And which perceived model is picked matters, because different perceived models will lead to different stabilization policies and thus different outcomes.

\subsection{The trade-off between the fiscal and monetary out- put responses}

Experts are left with only one degree of freedom in designing their model, which is captured by a trade-off between $\hat{a}$ and $\hat{b}$, the perceived effects on 
output of government spending and price expectations ${ }^{10}$. This trade-off is defined by the following formulae:

$$
\begin{aligned}
(\hat{b}-b) \omega \sigma_{u}^{2} & =\gamma[(\hat{a}-a)(\delta-\mu)+\hat{a} b-a \hat{b}] \\
\gamma & =-\hat{a} \frac{(\delta-\mu)^{2}}{\varphi(\delta+\hat{b}-\mu)^{2}+\hat{a}^{2}(\delta-\mu)^{2}} \omega \sigma_{u}^{2}
\end{aligned}
$$

Eliminating $\gamma$ between these two yields a cubic equation for $\hat{b}$, as a function of $\hat{a}$, which can be solved analytically, although numerical analysis is necessary to find out how $\hat{b}$ varies with $\hat{a}$ and the other parameters. Whenever there are three values of $\hat{b}$ that solve this equation, the largest root was selected. Given the requirement that $\hat{b}>0$, if that largest root is negative, then there is no plausible autocoherent model for this value of $\hat{a}$.

But much can be learned by considering the following approximation. Assume this is a "quasi-Lucas" economy, that is, $\delta-\mu<<1$. Then (14) is equivalent to

$$
\gamma \approx-\hat{a} \frac{(\delta-\mu)^{2}}{\varphi \hat{b}^{2}} \omega \sigma_{u}^{2}
$$

and substituting it into (13) we get

$$
\hat{b} \approx b-\frac{\hat{a}(\hat{a}-a)}{\varphi b}(\delta-\mu)^{2} .
$$

This trade-off has the following properties

\footnotetext{
${ }^{10}$ This degree of freedom comes from the fact that in this special case, one autocoherence condition becomes redundant. Thus one degree of freedom is left despite that the number of free parameters has been reduced to the number of autocoherence conditions.

Why is one autocoherence condition redundant here? Basically, if one only imposes that $\hat{\omega}=\omega, \hat{\sigma}_{u}=\sigma_{u}$, one can derive a condition involving $\hat{\delta}$ of which $\delta$ is a solution, although other values may also be solution in principle. Thus the condition $\hat{\delta}=\delta$ is almost endogenously derived from $\hat{\omega}=\omega, \hat{\sigma}_{u}=\sigma_{u}$. Imposing it rules out some other values of $\hat{\delta}$ but is redundant as long as $\delta$ is selected as the solution to the nonlinear equation which determines $\hat{\delta}$.
} 
- $(\hat{b}-b)(\hat{a}-a)<0$ and for $\hat{a}>a / 2, d \hat{b} / d \hat{a}<0$. Thus, the more the economist claims that government spending has a large impact on output, the lower the theoretical impact of interest rates. The only exception is if $\hat{a}$ is very low compared to $a$.

- The trade-off is flatter, the smaller $\delta-\mu$, the greater $\varphi$ and the greater $b$. That is, the more the government is averse to stabilization, the less favorable the Phillips curve, and the greater the true impact of interest rates, the more the theoretical effect of interest rates must be close to the actual one, and the more arbitrary the theoretical impact of government spending.

\subsection{Interpreting the autocoherence trade-off}

How can we make sense of these effects? In order to understand them we can focus on how $\hat{a}$ and $\hat{b}$ affect output's reaction to demand shocks, as captured by the value of $a_{y u}$ and its perceived counterpart

$$
\hat{a}_{y u}=-\frac{\hat{b}}{\hat{\delta}+\hat{b}-\hat{\mu}} \hat{\omega}^{2} \hat{\sigma}_{u}^{2}+\gamma \hat{\omega} \frac{\hat{a}(\hat{\delta}-\hat{\mu})}{\hat{\delta}+\hat{b}-\hat{\mu}}+1 .
$$

As stated above, autocoherence implies that the perceived model must correctly predict this elasticity. Furthermore, we also know that because of rational expectations this correct value only depends on the perceived model through the policy parameter $\gamma$. Consider an increase in $\hat{a}$ and hold $\gamma$ constant (the effect of the change in $\gamma$ is more complex and discussed in Remark 1 below). Then the equilibrium is unchanged and so is the output response $a_{y u}$. On the other hand, people will believe that $a_{y u}$ has fallen, since they think that the direct expansionary effect of fiscal policy (which outweighs its indirect contractionary effect through inflation expectations) is now stronger. This is captured by the fiscal component in $\hat{a}_{y u}, \gamma \hat{\omega} \frac{\hat{a}(\hat{\delta}-\hat{\mu})}{\hat{\delta}+\hat{b}-\hat{\mu}}$, which, since $\hat{\mu}<\hat{\delta}$ and $\gamma<0$, clearly falls in algebraic value as $\hat{a}$ goes up. This discrepancy 
would invalidate the model empirically unless $\hat{b}$ is changed so as to restore the equality between the actual and perceived elasticity of output to demand shocks. The dominant effect of a reduction in $\hat{b}$ (in a quasi-Lucas economy) is to increase the algebraic value of the perceived monetary component of $\hat{a}_{y u}$, given by $-\frac{\hat{b}}{\hat{\delta}+\hat{b}-\hat{\mu}} \hat{\omega}^{2} \hat{\sigma}_{u}^{2}{ }^{11}$ The lower $\hat{b}$, the lower the perceived output response to interest rates, and the lower the perceived stabilizing effect of monetary reactions to demand shocks. This effect raises the perceived response of output to demand shocks, thus restoring the model's autocoherence. This explains why there is a negative trade-off between $\hat{a}$ and $\hat{b}$. Since $\hat{b}$ is the interest elasticity of output, this means that experts face a trade-off between believing in fiscal policy effectiveness versus believing in monetary policy effectiveness. An economist who would underpredict both elasticities would also underpredict output volatility and could not empirically validate his model.

Remark 1: An increase in $\hat{a}$ also increases $\gamma$, the degree of fiscal activism. This magnifies the discrepancy between the perceived and actual fiscal components of $a_{y u}$-because government expenditures are more reactive to the demand shock signal. This discrepancy is negative if $\hat{a}>a$, i.e. people expect more fiscal stabilization than actually happens. In this case, the increase in $\gamma$ further widens the gap between actual and perceived fiscal components, thus reinforcing the negative required response of $\hat{b}$ to the increase in $\hat{a}$. On the other hand, if $\hat{a}<a$, the discrepancy is positive: people expect greater volatility of output coming from the fiscal component than in reality. While the direct effect of a greater $\hat{a}$ tends to make this discrepancy less positive, the indirect effect on $\gamma$ which magnifies the difference tends to make it larger. For $\hat{a}<a / 2$ this effect dominates, which explains why $d \hat{b} / d \hat{a}>0$ in this zone.

The size of the effects I just discussed is proportional to $|\gamma|$, the degree of fiscal activism. The lower $|\gamma|$, the lower the discrepancy between the actual

\footnotetext{
${ }^{11} \hat{b}$, also appears in the fiscal component but in a quasi-lucas economy this contribution is very small since that component is proportional to $(\hat{\delta}-\hat{\mu})^{3}$.
} 
and perceived fiscal components and the less reactive this discrepancy will be to an increase in $\hat{a}$. Thus, the less fiscal policy is active, the lower the deviation between $b$ and $\hat{b}$ that must be implemented to compensate a given deviation between $a$ and $\hat{a}$. In the limit case where $\gamma=0$, there is no variation is fiscal policy that would allow to identify $a$, and the only unidentified parameter that affects the output elasticity to demand shock is $b$, through the monetary component. Thus, in that limit case, $\hat{b}=b$ and $\hat{a}$ is arbitrary. In turn, fiscal activism is greater, the more favorable the output-inflation trade-off - the larger $\delta-\mu$-and the smaller the welfare cost $\varphi$ of fiscal volatility. This explains why the trade-off is flatter, the smaller $\delta-\mu$ and the greater $\varphi$.

Remark 2: Effect of $b$. The equilibrium output response $a_{y u}$ falls more with $\hat{a}$, the greater $b$. This is because the greater $b$, the greater the stabilizing effects of the monetary response to inflation. This reduces the reduction in $\hat{b}$ that is needed to offset an increase in $\hat{a}$, since the correct output response to demand shock that one has to match is now lower. Consequently, a greater value of $b$ makes the trade-off between $\hat{a}$ and $\hat{b}$ flatter.

Figure 1 depicts numerical simulations of the actual trade-off for four different sets of the parameters $a$ and $\delta-\mu$ (Note that the trade-off only depends on $\delta$ and $\mu$ through the difference $\delta-\mu) .{ }^{12}$ The results are very similar to what the above discussion based on the quasi-Lucas economy suggests. For $\hat{a}>a / 2$ the trade-off is decreasing and concave. It stops at a maximum value of $\hat{a}$ beyond which the plausibility condition $\hat{b}>0$ is violated. In most cases this corresponds to a catastrophe, mathematically speaking, in that the number of roots of the cubic equation defining $\hat{b}$ falls from 3 to 1 in such a way that the two largest roots disappear. Because of this discontinuity, the

\footnotetext{
${ }^{12}$ The other parameters in Figures 1 and 2 are $b=0.5, \varphi=0.8, \omega=1, \sigma_{u}^{2}=0.1, \sigma_{v}^{2}=$ 0.5 .
} 
curves on Figure 1 stop before hitting the horizontal axis. As in the quasiLucas case, it is flatter, the less favorable the Phillips curve, i.e. the smaller $\delta-\mu$. Furthermore, it shifts up and its slope becomes larger algebraically when $a$, the actual Keynesian multiplier, goes up, which is also implied by (16).

\subsection{The optimal model}

Which model will the expert select? As in Saint-Paul (2011), I assume his objective is $\bar{W}=\min \hat{E}\left(y^{2}+\bar{\varphi} g^{2}\right)$. In equilibrium, this is equal to (ignoring constants that are independent of the perceived model)

$$
\bar{W}=a_{y u}^{2} \sigma_{u}^{2}+a_{y \varepsilon}^{2} \sigma_{\varepsilon}^{2}+\bar{\varphi} \gamma^{2} .
$$

Since the reduced form elasticities $a_{y u}$ and $a_{y \varepsilon}$ only depend on the perceived model through $\gamma$, as long as the point chosen on the $(\hat{a}, \hat{b})$ trade-off is interior, the corresponding value of $\gamma$ is the one that would be obtained by directly maximizing $\bar{W}$ with respect to $\gamma$. In other words, unless plausibility constraints force him into an corner solution, the intellectual is a quasi-dictator, meaning that his preferred value of $\gamma$ is the one that would prevail if the intellectual were setting policy using the right model: ${ }^{13}$

$$
\gamma=\bar{\gamma}=-a \frac{(\delta-\mu)^{2}}{\bar{\varphi}(\delta+b-\mu)^{2}+a^{2}(\delta-\mu)^{2}} \omega \sigma_{u}^{2} .
$$

This equality allows us to find out how the perceived model depends on the economist's preferences. From this equality we have

$$
\frac{d \hat{a}}{d \bar{\varphi}}=\frac{\partial \bar{\gamma} / \partial \bar{\varphi}}{\partial \gamma / \partial \hat{a}+\partial \gamma / \partial \hat{b} \cdot d \hat{b} / d \hat{a}}
$$

\footnotetext{
${ }^{13}$ This can again be checked algebraically. The crucial autocoherence condition $\frac{\hat{\omega} \hat{\sigma}_{u}^{2}+\gamma \hat{a}}{\hat{\delta}+\hat{b}-\hat{\mu}}=\frac{\omega \sigma_{u}^{2}+\gamma a}{\delta+b-\mu}$ implies that $a_{y u}=\frac{\omega \gamma a(\delta-\mu)}{\delta+b-\mu}+1-\frac{b \omega^{2} \sigma_{u}^{2}}{\delta+b-\mu}$ and that $a_{y \varepsilon}=\frac{\gamma a(\delta-\mu)}{\delta+b-\mu}-\frac{b \omega \sigma_{u}^{2}}{\delta+b-\mu}$. Substituting these expressions into (17) and deriving the first-order conditions with respect to $\gamma$ delivers (18).
} 
where the derivative $d \hat{b} / d \hat{a}$ is taken along the autocoherence trade-off between $\hat{b}$ and $\hat{a}$. We know that $\partial \gamma / \partial \hat{b}>0, \partial \bar{\gamma} / \partial \bar{\varphi}>0$, and $\partial \gamma / \partial \hat{a}<0$ if the substitution effect dominates. Then, in the 'normal' part of the trade-off where $d \hat{b} / d \hat{a}<0$, we have that $\frac{d \hat{a}}{d \bar{\varphi}}<0$. More conservative economists will understate the impact of public expenditures and accordingly, to remain autocoherent, overstate that of interest rates. Furthermore, if the economist's preferences are aligned with that of the government, then the correct model is revealed, since by using it the government will then select $\gamma=\bar{\gamma}$. Since autocoherence imposes rational inflation expectations, there is no scope for manipulating the public and an economist aligned with the government cannot do better than reveal the truth.

Table 3 presents numerical simulations for various values of $\bar{\varphi}$, the degree of conservatism of the economist (the parameter values are the same as in Figure 1 and in particular $b=0.5$ ). It confirms that the more conservative the economist, the lower his theoretical IKM $\hat{a}$, and the larger the interest elasticity of output $\hat{b}$. Note also that a corner solution prevails for very progressive economists: the largest plausible value of $a$ is selected.

\begin{tabular}{|c|cc|cc|}
\hline \hline $\bar{\varphi}$ & \multicolumn{2}{|c|}{$a=0.2, \delta-\mu=0.4$} & \multicolumn{2}{c|}{$a=0.2, \delta-\mu=0.1$} \\
\hline & $\hat{a}$ & $\hat{b}$ & $\hat{a}$ & $\hat{b}$ \\
\cline { 2 - 5 } 0.08 & $1.1^{*}$ & 0.117 & 1.78 & 0.43 \\
0.4 & 0.39 & 0.48 & 0.34 & 0.498 \\
$0.8=\varphi$ & 0.2 & 0.5 & 0.2 & 0.5 \\
1.2 & 0.13 & 0.502 & 0.13 & 0.5 \\
1.6 & 0.1 & 0.502 & 0.1 & 0.5 \\
\hline \hline
\end{tabular}

\begin{tabular}{|cc|cc|}
\hline \hline \multicolumn{2}{|c|}{$a=0.8, \delta-\mu=0.4$} & \multicolumn{2}{|c|}{$a=0.8, \delta-\mu=0.1$} \\
\hline$\hat{a}$ & $\hat{b}$ & $\hat{a}$ & $\hat{b}$ \\
$1.48^{*}$ & 0.08 & $3.11^{*}$ & 0.21 \\
1.29 & 0.33 & 1.53 & 0.48 \\
0.8 & 0.5 & 0.8 & 0.5 \\
0.55 & 0.53 & 0.54 & 0.502 \\
0.42 & 0.534 & 0.4 & 0.503 \\
\hline
\end{tabular}


Table 3 -Ideological preferences and the expert's preferred perceived model.* $=$ maximum possible value.

\section{Manipulation of the Phillips curve parame- ters}

While economists' opinions about the Keynesian multiplier differ, so is the case with the parameters of the Phillips curve. But in the preceding model, these parameters cannot be manipulated in a way that matters for policy. ${ }^{14}$

I now study an example where the signal $z$ upon which forecasts are based does not allow to identify the slope of the Phillips curve $\delta-\mu$. That is, I assume that $z$ is now an aggregate of the demand and supply shock:

$$
z=\omega u-\lambda v
$$

Again I assume $\lambda, \omega>0$. The signal $z$ is interpreted as a signal about the aggregate price level. Thus this signal goes up with demand shocks but down with supply shocks. Furthermore, as the signal is polluted by the supply shock, it is no longer a valid instrument for estimating $\delta-\mu$. This quantity can no longer be inferred from the observed moments, and therefore autocoherence no longer compels the expert to reveal it.

I impose the following normalization:

$$
E\left(z^{2}\right)=\omega^{2} \sigma_{u}^{2}+\lambda^{2} \sigma_{v}^{2}=1
$$

To solve the model we now note that ${ }^{15} \hat{E}(u \mid z)=\hat{\omega} \hat{\sigma}_{u}^{2} z$ and $\hat{E}(v \mid$ $z)=-\hat{\lambda} \hat{\sigma}_{v}^{2} z$. Performing the same steps as in section 3.1 and using those

\footnotetext{
${ }^{14}$ Again that is not exactly true because $a$ and $b$ depend on $\delta$, so that the economist could distort $\delta$ in addition to $\alpha$ and $\beta$ to target $\hat{a}$ and $\hat{b}$, despite that this extra degree of freedom is not needed.

${ }^{15}$ This again anticipates on the autocoherence condition $E\left(z^{2}\right)=\hat{E}\left(z^{2}\right)=1$.
} 
expressions, we get that

$$
p^{e}=\frac{\hat{a}}{\hat{\delta}+\hat{b}-\hat{\mu}} g+\hat{c} z
$$

with

$$
\hat{c}=\frac{\hat{\omega} \hat{\sigma}_{u}^{2}-\hat{\lambda}(\hat{\rho}-1) \hat{\sigma}_{v}^{2}}{\hat{\delta}+\hat{b}-\hat{\mu}} .
$$

Therefore the solution is

$$
\begin{gathered}
y=u+\rho v-b \hat{c} z+\left(a-\frac{b \hat{a}}{\hat{\delta}+\hat{b}-\hat{\mu}}\right) g \\
p=\frac{\mu-b}{\delta} \hat{c} z+\left(\frac{a}{\delta}+\frac{\hat{a}(\mu-b)}{\delta(\hat{\delta}+\hat{b}-\hat{\mu})}\right) g+\frac{u}{\delta}+\frac{\rho-1}{\delta} v .
\end{gathered}
$$

How is government policy determined in this variant of the model? Conditions (7) and (8) as well as the FOC (6) still hold. But applying hatted expectations to both sides of (19) we now get

$$
\hat{E}(y \mid z)=\frac{\hat{a}(\hat{\delta}-\hat{\mu})}{\hat{\delta}+\hat{b}-\hat{\mu}} g+\left[\hat{\omega} \hat{\sigma}_{u}^{2} \frac{\hat{\delta}-\hat{\mu}}{\hat{\delta}+\hat{b}-\hat{\mu}}-\hat{\lambda} \hat{\sigma}_{v}^{2}\left(\hat{\rho}-\frac{(\hat{\rho}-1) \hat{b}}{\hat{\delta}+\hat{b}-\hat{\mu}}\right)\right] z
$$

Consequently optimal fiscal policy is now given by $g=\gamma z$, with

$$
\gamma=\hat{a} \frac{\hat{\lambda} \hat{\sigma}_{v}^{2}\left[\hat{\rho}(\hat{\delta}-\hat{\mu})^{2}+\hat{b}(\hat{\delta}-\hat{\mu})\right]-(\hat{\delta}-\hat{\mu})^{2} \hat{\omega} \hat{\sigma}_{u}^{2}}{\varphi(\hat{\delta}+\hat{b}-\hat{\mu})^{2}+\hat{a}^{2}(\hat{\delta}-\hat{\mu})^{2}} \lessgtr 0 .
$$

Note that the sign of $\gamma$ depends on the relative importance of supply and demand shocks. If supply shocks are perceived to be more important $\left(\hat{\sigma}_{v}^{2}\right.$ large enough relative to $\left.\hat{\sigma}_{u}^{2}\right)$, an indication of price pressure $(z>0)$ signals a contraction and will be met with expansionary policies $(\gamma>0) .{ }^{16}$

\footnotetext{
${ }^{16}$ Clearly, this could change if a price stability objective were added to the government's utility function.
} 
The model's new solution is now given in Tables 4 and 5 .

\begin{tabular}{ll}
\hline \hline Observable & Expression \\
\hline Output & $y=a_{y u} u+a_{y v} v$ \\
Price & $p=a_{p u} u+a_{p v} v$ \\
\hline \hline Coefficients & Expression \\
\hline$a_{y u}$ & $1-b \omega \hat{c}+\omega \gamma\left(a-\frac{\hat{a} b}{\hat{\delta}+\hat{b}-\hat{\mu}}\right)$ \\
$a_{y v}$ & $\rho+b \lambda \hat{c}-\gamma \lambda\left(a-\frac{\hat{a} b}{\hat{\delta} \hat{b}-\hat{\mu}}\right)$ \\
$a_{p u}$ & $\omega \gamma\left(\frac{a}{\delta}+\frac{\hat{a}(\mu-b)}{\delta(\hat{\delta}+\hat{b}-\hat{\mu})}\right)+(\mu-b) \frac{\omega \hat{c}}{\delta}+\frac{1}{\delta}$ \\
$a_{p v}$ & $\frac{\rho-1}{\delta}-(\mu-b) \frac{\lambda \hat{c}}{\delta}-\lambda \gamma\left(\frac{a}{\delta}+\frac{\hat{a}(\mu-b)}{\delta(\hat{\delta}+\hat{b}-\hat{\mu})}\right)$. \\
\hline \hline
\end{tabular}

$\overline{\text { Table } 4 \text { - The correct reduced form model, Variant B }}$

\begin{tabular}{ll}
\hline \hline Observable & Expression \\
\hline Output & $y=\hat{a}_{y u} \hat{u}+\hat{a}_{y v} \hat{v}$ \\
Price & $p=\hat{a}_{p u} \hat{u}+\hat{a}_{p v} \hat{v}$ \\
\hline \hline Coefficients & Expression \\
\hline$\hat{a}_{y u}$ & $1-\hat{b} \hat{\omega} \hat{c}+\hat{\omega} \gamma \frac{\hat{a}(\hat{\delta}-\hat{\mu})}{\hat{\delta}+\hat{b}-\hat{\mu}}$ \\
$a_{y v}$ & $\hat{\rho}+\hat{b} \hat{\lambda} \hat{c}-\gamma \hat{\lambda} \frac{\hat{a} \hat{\delta}-\hat{\mu})}{\hat{\delta}+\hat{b}-\hat{\mu}}$ \\
$a_{p u}$ & $\hat{\omega} \gamma_{\frac{\hat{\sigma}}{\hat{\delta}+\hat{b}-\hat{\mu}}}+(\hat{\mu}-\hat{b}) \frac{\hat{\omega} \hat{c}}{\hat{\delta}}+\frac{1}{\hat{\delta}}$ \\
$a_{p v}$ & $\frac{\hat{\rho}-1}{\hat{\delta}}-(\hat{\mu}-\hat{b}) \frac{\hat{\lambda} \hat{c}}{\hat{\delta}}-\hat{\lambda} \gamma_{\frac{\hat{\alpha}}{\hat{\delta}+\hat{b}-\hat{\mu}}}$ \\
\hline
\end{tabular}

Table 5 - The perceived reduced form model, Variant B

There are again six autocoherence solutions and nine parameters. In contrast to the previous section, I will now assume that the key parameters of the output block are common knowledge: $\hat{a}=a$ and $\hat{b}=b$. The autocoherence conditions now leave us with one degree of freedom: they define a 1-dimensional manifold in a 7-dimensional space. Rather than solving those highly nonlinear equations, I linearize the system of autocoherence conditions locally around the correct model. For such "quasi-correct" models, the autocoherence conditions are thus a straight line in that space. Define $\Delta \hat{\delta}=\hat{\delta}-\delta<<1$ and similarly for other parameters. Then we can reexpress 
the $\mathrm{AC}$ conditions in the following fashion

$$
v=\Delta \hat{\delta} \cdot q
$$

where $v=\left(\Delta(\hat{\delta}-\hat{\mu}), \Delta \hat{\lambda}, \Delta \hat{\sigma}_{v}, \Delta \hat{\omega}, \Delta \hat{\sigma}_{u}, \Delta \hat{\rho}\right)^{\prime}$ and $q$ is a 6-dimensional vector whose $i$ th coefficient gives us the slope of the trade-off between $\hat{\delta}$ and the $i$ th parameter in $v .{ }^{17}$ Of special interest is the first coefficient of $q$ since it defines the set of slope parameters of the long-run Phillips curve that the economist may promote to influence policy while remaining autocoherent.

The algebraic steps to derive the $q$ vector are described in the Appendix, and these formulas can be used to numerically compute $q$ in a given economy.

Which point is going to be selected by the economist along this autocoherence locus? Again, he will be a quasi-dictator and it is natural, given our approximation, to assume that his preferences differ only marginally from those of the government: $\bar{\varphi}=\varphi+\Delta \varphi, \Delta \varphi<<1$. Let $\gamma_{0}$ be the value of $\gamma$ prevailing if the perceived model is correct, then the target value of $\gamma$ for the economist is given by $\tilde{\gamma} \approx \gamma_{0}+\frac{\partial \gamma}{\partial \varphi} \Delta \varphi=\gamma_{0}+\Delta \tilde{\gamma}$. On the other hand, the value of $\gamma$ pursued by the government given the perceived model can be expressed as $\gamma \approx \gamma_{0}+\left(\nabla_{v} \gamma\right) \cdot v=\gamma_{0}+\Delta \gamma$, where $\nabla_{v} \gamma$ is the appropriate vectors of derivatives ${ }^{18}$. They and $\frac{\partial \gamma}{\partial \varphi}$ are computed in the Appendix. The economist will pick the model that satisfies $\Delta \gamma=\Delta \tilde{\gamma}$, implying that the perceived model can be summarized by a relationship between $\Delta \hat{\delta}$ and $\Delta \varphi$ :

$$
\Delta \hat{\delta}=m \Delta \varphi
$$

where

$$
m=\frac{\frac{\partial \gamma}{\partial \varphi}}{\left(\nabla_{v} \gamma\right) \cdot q}
$$

\footnotetext{
${ }^{17}$ Given the particular importance of the parameter $\delta-\mu$, I prefer to use $\hat{\delta}-\hat{\mu}$ rather than $\hat{\mu}$.

${ }^{18}$ There is no contribution of $\Delta \hat{\delta}$ in the differentiation $\gamma$ with respect to the perceived parameters once one also differentiates with respect to the parameters in $v$, since $\hat{\delta}$ only appears through $\hat{\delta}-\hat{\mu}$.
} 
I will now use those results to analyze the structure of the perceived model and how it depends on the underlying parameters of the economy. In order to organize the discussion, I will focus on five intuitive characteristics of a theory:

1. The short-term inflationary cost of output (STC). This is equal to $1 / \hat{\delta}$.

2. The long-term inflationary cost of output (LTC), equal to $1 /(\hat{\delta}-\hat{\mu})$.

3. The relative importance of supply shocks (RIS), equal to $\frac{\hat{\sigma}_{v}^{2}}{\hat{\sigma}_{u}^{2}}$.

4. The supply-intensity of the price indicator (SIP), equal to $\hat{\lambda}^{2} \hat{\sigma}_{v}^{2}$.

5. The share of output fluctuations explained by supply shocks (SSO); given by $\frac{\hat{a}_{y v}^{2} \hat{\sigma}_{v}^{2}}{\hat{a}_{y v}^{2} \hat{\sigma}_{v}^{2}+\hat{a}_{y y u}^{2} \hat{\sigma}_{u}^{2}}$.

For each of these parameters, its ideological sensitivity is defined as its derivative with respect to $\varphi$. A positive ideological sensitivity means that the parameter goes up, the more conservative the economist. The greater the absolute value of ideological sensitivity, the more the parameter will deviate from its true value as a result of the economist's own agenda (and, intuitively, we expect economists with different ideological positions to disagree more about that parameter). The expressions for the ideological sensitivities are given by the following Table.

\begin{tabular}{ll}
\hline \hline Parameter & Ideological sensitivity \\
\hline STC & $-m / \delta^{2}$ \\
LTC & $-m q_{1} /(\delta-\mu)^{2}$ \\
RIS & $\frac{2 \sigma_{v}}{\sigma_{u}^{2}} m\left(q_{3}-\frac{\sigma_{v}}{\sigma_{u}} q_{5}\right)$ \\
SIP & $2 m\left(\lambda \sigma_{v}^{2} q_{2}+\lambda^{2} \sigma_{v} q_{3}\right)$ \\
\hline \hline SSO & See Appendix \\
\hline \hline
\end{tabular}

Table 6 - Ideological sensitivities of key perceived parameters

Figures 4 to 9 report ideological sensitivities, as $\mu$ varies, for 5 sets of values for the other parameters. We observe the following: 
- Typically, the ideological sensitivity of LTC is positive: more conservative economists will report a higher inflationary cost of output in the long run. This makes sense as it will deter activist stabilization policies. However, there are exceptions: on Figure 9 where $b$ is quite low ( $b=0.1$ ), LTC has a positive sensitivity only if $\mu$ is large enough, i.e. on the right of the asymptote.

- However, for other parameters, things are less clear-cut. For example, the STC is always negative except on Figure 9. A conservative economist wants to downplay the efficiency of stabilization through public expenditures, but cannot act on all margins simultaneously because he is bound by the autocoherence conditions. This sometimes forces him to appear progressive on some fronts, as is the case for the short-term inflationary effects of inflation.

- Nevertheless, a pattern emerges: the ideological sensitivity of STC is always small, implying that the truth is almost revealed about $\delta$ regardless of the economist's ideological position, while there is much more ideological polarization with respect to the value of $\mu$. A conservative economist will overemphasize the negative impact of inflationary expectations on output, in a way reminiscent of Friedman (1968) and Lucas $(1972,1973)$, while the left-wing economist will produce models that understate $\mu$, in a fashion not unlike that of Akerlof, Dickens and Perry (2000).

- We also note that in many simulations the share of output fluctuations explained by supply shocks has a positive ideological sensitivity - conservative economists will predict that supply shocks play a bigger role in output fluctuations; however this does not happen because of the RIS, which tends to have a negative sensitivity, but through the per- 
ceived responses of output to these shocks $\hat{a}_{y u}$ and $\hat{a}_{y v}$. An exception arises when $\sigma_{u}$ is very large (Figure 5 ), or $b$ very low (Figure 9). In Figure 9, the conservative economist believes in a mildly more favorable Phillips curve for $\mu$ low, but also promotes the view that supply shocks are relatively important. If $\mu$ is high, the pattern is similar to the other figures.

- An economy can be "critical", meaning that the denominator of (21) is close to zero. This happens on Figure 9 around $\mu \approx 0.59$, and on Figure 5 around $\mu=0.66$. In a critical economy, parameters happen to be such that ideology is uninfluential. To compensate for that and act as quasi-dictators, economists will tend to pick very large deviations between the perceived and actual parameters: ideological sensitivities become very large, as captured by the asymptotes in our figures. This in fact means that our approximation is no longer valid; still small ideological deviations have large effects on the prevailing view of the world. Intuitively, an economy is critical if parameter values are such that the autocoherence locus (a manifold in the perceived parameters space) is included in an iso-policy locus. Here, this is true locally: the vector $q$, which tells us the direction where the perceived model must move to remain autocoherent, is orthogonal to the policy gradient $\nabla_{v} \gamma$, and therefore autocoherence implies local policy invariance. Note, however, that the result that ideological deviations become large around a critical economy would be overturned if there was some convex cost to the expert of deviating from the truth; economists would then no longer be quasi-dictators and in a critical economy, the benefits of manipulation would be negligible relative to the costs of deviating from the truth. Instead of becoming infinite, ideological sensitivities would then fall to 
zero in a critical economy ${ }^{19}$.

\section{Conclusion}

Admittedly the present model does not have tight empirical predictions. The equilibrium perceived model that one expects is highly dependent on the correct model, the set of variables that are observable, and the set of parameters that are common knowledge. Nevertheless, this framework allows to identify the set of "credible" model parameters and to give some insight about how political preferences will affect the choice of those parameters. In particular, key parameters will be self-serving in the "expected" way, but this typically implies that concessions have to be made with respect to the other parameters. The analysis has also elicited the phenomenon of criticality, i.e. economies where ideologies are ineffective, and for that reason tend to be exacerbated.

\footnotetext{
${ }^{19}$ This can be seen by looking at the following reduced form optimization problem: $\min _{\hat{\theta}} c(\hat{\theta}-\theta)^{2}+(s \hat{\theta}-\varphi)^{2}$, where $\theta$ is the true parameter value, $\hat{\theta}$ the perceived one, $s \hat{\theta}$ the outcome (up to a constant), $\varphi$ the target outcome, and $c$ the cost of deviating from the truth. The optimal value of $\hat{\theta}$ is $\frac{c \theta+s \varphi}{c+s^{2}}$, with a radically different behavior around $s=0$ (criticality) depending on whether $c$ is positive vs. zero.
} 


\section{REFERENCES}

Akerlof George A., William T. Dickens, and George L. Perry, "NearRational Wage and Price Setting and the Long-Run Phillips Curve", Brookings Papers on Economic Activity, Vol. 2000, No. 1 (2000), pp. 1-60

Friedman, Milton (1968), "he Role of Monetary Policy", The American Economic Review, 58, 1, 1-17

Fuchs, Victor, Alan Krueger and James M. Porterba (1998), "Economists' views about parameters, values, and policies: Survey results in labor and public economics", Journal of Economic Literature, 36, 3, 1387-1425

Fudenberg. D. and D.K. Levine (1993) "Self-Confirming Equilibrium" Econometrica, 61 , 523-546.

(2007), "Self-confirming equilibrium and the Lucas critique"

Lucas, Robert (1972), "Expectations and the neutrality of money", Journal of Economic Theory

- (1973), "International evidence on the output-inflation trade-off", American Economic Review

Saint-Paul, Gilles (2011), "Toward a Political Economy of Macroeconomic Thinking"

Sargent, Thomas (2008), "Evolution and Intelligent Design", American Economic Review, 98(1), 5-37 


\section{APPENDIX}

\subsection{Derivation of the autocoherence conditions}

1. Variance of $z$

$$
\begin{aligned}
E z^{2} & =1 \\
& =\omega^{2} \sigma_{u}^{2}+\sigma_{\varepsilon}^{2} \\
& =\hat{E} z^{2} \\
& =\hat{\omega}^{2} \hat{\sigma}_{u}^{2}+\hat{\sigma}_{\varepsilon}^{2} .
\end{aligned}
$$

2. Covariance between $z$ and $y$

$$
\begin{aligned}
E y z & =a_{y u} \omega \sigma_{u}^{2}+a_{y \varepsilon} \sigma_{\varepsilon}^{2} \\
& =\hat{E} y z \\
& =\hat{a}_{y u} \hat{\omega} \hat{\sigma}_{u}^{2}+\hat{a}_{y \varepsilon} \hat{\sigma}_{\varepsilon}^{2}
\end{aligned}
$$

Using (22), (23) can be rewritten

$$
\begin{aligned}
\left(a_{y \varepsilon} \omega+1\right) \omega \sigma_{u}^{2}+a_{y \varepsilon}\left(1-\omega^{2} \sigma_{u}^{2}\right) & =\left(\hat{a}_{y \varepsilon} \hat{\omega}+1\right) \hat{\omega} \hat{\sigma}_{u}^{2}+\hat{a}_{y \varepsilon}\left(1-\hat{\omega}^{2} \hat{\sigma}_{u}^{2}\right) \\
& \Longleftrightarrow \\
\omega \sigma_{u}^{2}+a_{y \varepsilon} & =\hat{\omega} \hat{\sigma}_{u}^{2}+\hat{a}_{y \varepsilon}
\end{aligned}
$$

3. Covariance between $z$ and $p$

$$
\begin{aligned}
E p z & =a_{p u} \omega \sigma_{u}^{2}+a_{p \varepsilon} \sigma_{\varepsilon}^{2} \\
& =\hat{E} p z \\
& =\hat{a}_{p u} \hat{\omega} \hat{\sigma}_{u}^{2}+\hat{a}_{p \varepsilon} \hat{\sigma}_{\varepsilon}^{2}
\end{aligned}
$$


Using similar steps as above, we can see that this is equivalent to

$$
\frac{\omega \sigma_{u}^{2}}{\delta}+a_{p \varepsilon}=\frac{\hat{\omega} \hat{\sigma}_{u}^{2}}{\hat{\delta}}+\hat{a}_{p \varepsilon} .
$$

4. Covariance between $y$ and $p$

$$
\begin{aligned}
E p y & =a_{y u} a_{p u} \sigma_{u}^{2}+a_{y \varepsilon} a_{p \varepsilon} \sigma_{\varepsilon}^{2}+\frac{\rho(\rho-1)}{\delta} \sigma_{v}^{2} \\
& =\left(a_{y \varepsilon} \omega+1\right)\left(\frac{1}{\delta}+\omega a_{p \varepsilon}\right) \sigma_{u}^{2}+a_{y \varepsilon} a_{p \varepsilon} \sigma_{\varepsilon}^{2}+\frac{\rho(\rho-1)}{\delta} \sigma_{v}^{2} \\
& =\left(\frac{1}{\delta}+\omega a_{p \varepsilon}+a_{y \varepsilon} \omega\right) \sigma_{u}^{2}+a_{y \varepsilon} a_{p \varepsilon}+\frac{\rho(\rho-1)}{\delta} \sigma_{v}^{2} \\
& =\hat{E} p y \\
& =\left(\frac{1}{\hat{\delta}}+\hat{\omega} \hat{a}_{p \varepsilon}+\hat{a}_{y \varepsilon} \hat{\omega}\right) \hat{\sigma}_{u}^{2}+\hat{a}_{y \varepsilon} \hat{a}_{p \varepsilon}+\frac{\hat{\rho}(\hat{\rho}-1)}{\hat{\delta}} \hat{\sigma}_{v}^{2} .
\end{aligned}
$$

5. Variance of $y$

$$
\begin{aligned}
E y^{2} & =a_{y u}^{2} \sigma_{u}^{2}+a_{y \varepsilon}^{2} \sigma_{\varepsilon}^{2}+\rho^{2} \sigma_{v}^{2} \\
& =\left(a_{y \varepsilon} \omega+1\right)^{2} \sigma_{u}^{2}+a_{y \varepsilon}^{2}\left(1-\omega^{2} \sigma_{\varepsilon}^{2}\right)+\rho^{2} \sigma_{v}^{2} \\
& =\left(1+2 a_{y \varepsilon} \omega\right) \sigma_{u}^{2}+a_{y \varepsilon}^{2}+\rho^{2} \sigma_{v}^{2} \\
& =\hat{E} y^{2} \\
& =\left(1+2 \hat{a}_{y \varepsilon} \hat{\omega}\right) \hat{\sigma}_{u}^{2}+\hat{a}_{y \varepsilon}^{2}+\hat{\rho}^{2} \hat{\sigma}_{v}^{2} .
\end{aligned}
$$

6. Variance of $p$.

Note that this autocoherence condition can always be matched by picking the right value of $\hat{\sigma}_{\eta}^{2}$, regardless of the other parameters of the perceived model. I write it for the record. 


$$
\begin{aligned}
E p^{2} & =a_{p u}^{2} \sigma_{u}^{2}+a_{p \varepsilon}^{2} \sigma_{\varepsilon}^{2}+\frac{(\rho-1)^{2}}{\delta^{2}} \sigma_{v}^{2}+\sigma_{\eta}^{2} \\
& =\left(\frac{1}{\delta^{2}}+\frac{2 a_{p \varepsilon} \omega}{\delta}\right) \sigma_{u}^{2}+a_{p \varepsilon}^{2}+\frac{(\rho-1)^{2}}{\delta^{2}} \sigma_{v}^{2} \\
& =\hat{E} p^{2} \\
& =\left(\frac{1}{\hat{\delta}^{2}}+\frac{2 \hat{a}_{p \varepsilon} \hat{\omega}}{\hat{\delta}}\right) \hat{\sigma}_{u}^{2}+\hat{a}_{p \varepsilon}^{2}+\frac{(\hat{\rho}-1)^{2}}{\hat{\delta}^{2}} \hat{\sigma}_{v}^{2}
\end{aligned}
$$

\subsection{Proof of Proposition 1}

As proved in footnote 8, we know that condition (24) is equivalent to

$$
\frac{\hat{\omega} \hat{\sigma}_{u}^{2}+\gamma \hat{a}}{\hat{\delta}+\hat{b}-\hat{\mu}}=\frac{\omega \sigma_{u}^{2}+\gamma a}{\delta+b-\mu}
$$

Using the definition of $a_{y \varepsilon}$ and $\hat{a}_{y \varepsilon}$, we can rewrite (23) as

$$
\omega \sigma_{u}^{2}+\gamma a-b\left(\frac{\hat{\omega} \hat{\sigma}_{u}^{2}+\gamma \hat{a}}{\hat{\delta}+\hat{b}-\hat{\mu}}\right)=\hat{\omega} \hat{\sigma}_{u}^{2}+\gamma \hat{a}-\hat{b}\left(\frac{\hat{\omega} \hat{\sigma}_{u}^{2}+\gamma \hat{a}}{\hat{\delta}+\hat{b}-\hat{\mu}}\right)
$$

Replacing $\hat{\omega} \hat{\sigma}_{u}^{2}+\gamma \hat{a}$ with $\frac{\omega \sigma_{u}^{2}+\gamma a}{\delta+b-\mu}(\hat{\delta}+\hat{b}-\hat{\mu})$ and rearranging, we indeed get $\hat{\delta}-\hat{\mu}=\delta-\mu$.

QED

\subsection{The price block revealed case}

Assume $\hat{\omega}=\omega, \hat{\sigma}_{u}=\sigma_{u}$, and $\hat{\delta}=\delta$. We know from Proposition 2 that $\hat{\mu}=\mu$. From (22) we get

$$
\hat{\sigma}_{\varepsilon}^{2}=\sigma_{\varepsilon}^{2} .
$$

From (23) we get 


$$
a_{y \varepsilon}=\hat{a}_{y \varepsilon},
$$

Similarly, for (24) to hold we need

$$
a_{p \varepsilon}=\hat{a}_{p \varepsilon}
$$

This in turn implies $a_{y u}=\hat{a}_{y u}$ and $a_{p u}=\hat{a}_{p u}$.

Finally, (25) and (26) yield

$$
\begin{aligned}
\frac{\rho(\rho-1)}{\delta} \sigma_{v}^{2} & =\frac{\hat{\rho}(\hat{\rho}-1)}{\hat{\delta}} \hat{\sigma}_{v}^{2} \\
\hat{\rho}^{2} \hat{\sigma}_{v}^{2} & =\rho^{2} \sigma_{v}^{2}
\end{aligned}
$$

The solution to this system is

$$
\begin{aligned}
\rho & =\hat{\rho} ; \\
\hat{\sigma}_{v}^{2} & =\sigma_{v}^{2} .
\end{aligned}
$$

From (28) we get

$$
\omega \sigma_{u}^{2}(\hat{b}-b)=\gamma[\hat{a}(\delta+b-\mu)-a(\hat{\delta}+\hat{b}-\hat{\mu})] .
$$

Recall, from (11), that

$$
\gamma=-\hat{a} \frac{(\delta-\mu)^{2}}{\varphi(\delta+\hat{b}-\mu)^{2}+\hat{a}^{2}(\delta-\mu)^{2}} \omega \sigma_{u}^{2} .
$$

Substituting, we get the cubic equation that has been solved numerically:

$(\hat{b}-b)\left(\varphi(\delta+\hat{b}-\mu)^{2}+\hat{a}^{2}(\delta-\mu)^{2}\right)+\hat{a}(\delta-\mu)^{2}[(\hat{a}-a)(\delta-\mu)+\hat{a} b-a \hat{b}]=0$.

Finally, the above conditions trivially imply that the remaining condition (27) holds. 


\subsection{Linearization of the $\mathrm{AC}$ conditions in variant $\mathrm{B}$}

The six AC conditions are

$$
\begin{aligned}
1 & =\hat{\omega}^{2} \hat{\sigma}_{u}^{2}+\hat{\lambda}^{2} \hat{\sigma}_{v}^{2} ; \\
a_{y u} \omega \sigma_{u}^{2}-a_{y v} \lambda \sigma_{v}^{2} & =\hat{a}_{y u} \hat{\omega} \hat{\sigma}_{u}^{2}-\hat{a}_{y v} \hat{\lambda} \hat{\sigma}_{v}^{2} ; \\
a_{p u} \omega \sigma_{u}^{2}-a_{p v} \lambda \sigma_{v}^{2} & =\hat{a}_{p u} \hat{\omega} \hat{\sigma}_{u}^{2}-\hat{a}_{p v} \hat{\lambda} \hat{\sigma}_{v}^{2} ; \\
a_{y u} a_{p u} \sigma_{u}^{2}+a_{y v} a_{p v} \sigma_{v}^{2} & =\hat{a}_{y u} \hat{a}_{p u} \hat{\sigma}_{u}^{2}+\hat{a}_{y v} \hat{a}_{p v} \hat{\sigma}_{v}^{2} ; \\
a_{y u}^{2} \sigma_{u}^{2}+a_{y v}^{2} \sigma_{v}^{2} & =\hat{a}_{y u}^{2} \hat{\sigma}_{u}^{2}+\hat{a}_{y v}^{2} \hat{\sigma}_{v}^{2} ; \\
a_{p u}^{2} \sigma_{u}^{2}+a_{p v}^{2} \sigma_{v}^{2} & =\hat{a}_{p u}^{2} \hat{\sigma}_{u}^{2}+\hat{a}_{p v}^{2} \hat{\sigma}_{v}^{2} .
\end{aligned}
$$

Using the definitions in Table 4 and 5 to rearrange (35), and defining $c=$ $\frac{\omega \sigma_{u}^{2}-\lambda(\rho-1) \sigma_{v}^{2}}{\delta+b-\mu}$, we see that (35) is equivalent to

$$
\hat{c}+\frac{\gamma \hat{a}}{\hat{\delta}+\hat{b}-\hat{\mu}}=c+\frac{\gamma a}{\delta+b-\mu} .
$$

This expression can be conveniently substituted into the expressions in Tables 4 and 5 to reduce the number of hatted parameters that appear. We get the following:

\begin{tabular}{ll}
\hline \hline Coefficients & Expression \\
\hline$a_{y u}$ & $1+\omega \gamma a-b \omega\left(c+\frac{\gamma a}{\delta+b-\mu}\right)$ \\
$a_{y v}$ & $\rho-\gamma \lambda a+b \lambda\left(c+\frac{\gamma a}{\delta+b-\mu}\right)$ \\
$a_{p u}$ & $\frac{1}{\delta}+(\mu-b) \frac{\omega}{\delta}\left(c+\frac{\gamma a}{\delta+b-\mu}\right)+\frac{\gamma a \omega}{\delta}$ \\
$a_{p v}$ & $\frac{\rho-1}{\delta}-(\mu-b) \frac{\lambda}{\delta}\left(c+\frac{\gamma a}{\delta+b-\mu}\right)-\frac{\gamma a \lambda}{\delta}$ \\
$\hat{a}_{y u}$ & $1+\hat{\omega} \gamma \hat{a}-\hat{b} \hat{\omega}\left(c+\frac{\gamma a}{\delta+b-\mu}\right)$ \\
$\hat{a}_{y v}$ & $\hat{\rho}-\gamma \hat{\lambda} \hat{a}+\hat{b} \hat{\lambda}\left(c+\frac{\gamma a}{\delta+b-\mu}\right)$ \\
$\hat{a}_{p u}$ & $\frac{1}{\hat{\delta}}+(\hat{\mu}-\hat{b}) \frac{\hat{\omega}}{\hat{\delta}}\left(c+\frac{\gamma a}{\delta+b-\mu}\right)+\frac{\gamma \hat{a} \hat{\omega}}{\hat{\delta}}$ \\
$\hat{a}_{p v}$ & $\frac{\hat{\rho}-1}{\hat{\delta}}-(\hat{\mu}-\hat{b}) \frac{\hat{\lambda}}{\hat{\delta}}\left(c+\frac{\gamma a}{\delta+b-\mu}\right)-\frac{\gamma \hat{a} \hat{\lambda}}{\hat{\delta}}$ \\
\hline \hline
\end{tabular}


From now on we will take into account that $\hat{a}=a$ and $\hat{b}=b$. Using this Table we can then compute $\Delta \hat{a}_{y u}=\hat{a}_{y u}-a_{y u}$, etc. ${ }^{20}$ We get

$$
\begin{aligned}
\Delta \hat{a}_{y u} \approx & \left(\frac{\gamma a(\delta-\mu)}{\delta+b-\mu}-b c\right) \Delta \hat{\omega} ; \\
\Delta \hat{a}_{p u} \approx & -\frac{\Delta \hat{\delta}}{\delta^{2}}\left(1+(\mu-b) \omega c+\frac{\gamma a \omega \delta}{\delta+b-\mu}\right)+\frac{\Delta \hat{\mu}}{\delta} \omega\left(c+\frac{\gamma a}{\delta+b-\mu}\right) \\
& +\frac{\Delta \hat{\omega}}{\delta}\left((\mu-b) c+\frac{\gamma a \delta}{\delta+b-\mu}\right) ; \\
\Delta \hat{a}_{y v} \approx & \Delta \hat{\rho}+\Delta \hat{\lambda}\left(b c-\frac{\gamma a(\delta-\mu)}{\delta+b-\mu}\right) ; \\
\Delta \hat{a}_{p v} \approx & \frac{\Delta \hat{\rho}}{\delta}-\frac{\Delta \hat{\delta}}{\delta^{2}}\left(\rho-1-(\mu-b) \lambda c-\frac{\gamma a \lambda \delta}{\delta+b-\mu}\right) \\
& -\frac{\Delta \hat{\mu}}{\delta} \lambda\left(c+\frac{\gamma a}{\delta+b-\mu}\right)-\frac{\Delta \hat{\lambda}}{\delta}\left((\mu-b) c+\frac{\gamma a \delta}{\delta+b-\mu}\right) .
\end{aligned}
$$

We can also compute

$$
\begin{aligned}
\Delta \hat{c} \approx & -\frac{\omega \sigma_{u}^{2}-\lambda(\rho-1) \sigma_{v}^{2}}{(\delta+b-\mu)^{2}}(\Delta \hat{\delta}-\Delta \hat{\mu}) \\
& +\frac{1}{\delta+b-\mu}\left[\sigma_{u}^{2} \Delta \hat{\omega}+2 \omega \sigma_{u} \Delta \hat{\sigma}_{u}-\lambda \sigma_{v}^{2} \Delta \hat{\rho}-(\rho-1) \sigma_{v}^{2} \Delta \hat{\lambda}-2 \lambda(\rho-1) \sigma_{v} \Delta \hat{\sigma}_{v}\right]
\end{aligned}
$$

Finally, substituting (39) into (34) and rearranging using the definitions in Tables 4 and 5 we get the following:

$$
\left(c+\frac{\gamma a}{\delta+b-\mu}\right)[\hat{\delta}-\hat{\mu}-\delta+\mu]=\hat{\lambda} \hat{\sigma}_{v}^{2}-\lambda \sigma_{v}^{2} .
$$

Next, we differentiate (33)-(38), substituting (39) and (40) for (35) and (34) respectively, and replacing $\Delta \hat{a}_{y u}$,etc., as well as $\Delta \hat{c}$ by their expressions above. We get six linear equations that are expressed as

\footnotetext{
${ }^{20}$ Note that a small deviation between the perceived and correct model changes $\gamma$ marginally, hence $a_{y u}$ is different from its value under the correct model, and thus $\Delta \hat{a}_{y u}$ is not equal to the difference between $\hat{a}_{y u}$ and the value of $a_{y u}$ under the correct model.
} 


$$
A .\left(\Delta(\hat{\delta}-\hat{\mu}), \Delta \hat{\lambda}, \Delta \hat{\sigma}_{v}, \Delta \hat{\omega}, \Delta \hat{\sigma}_{u}, \Delta \hat{\rho}\right)^{\prime}=\Delta \hat{\delta} . w,
$$

where the nonzero coefficients of $A: 6 \times 6$, and $w: 6 \times 1$ are the following:

$$
\begin{aligned}
& A_{12}=\lambda \sigma_{v}^{2} ; A_{13}=\lambda^{2} \sigma_{v} ; A_{14}=\omega \sigma_{u}^{2} ; A_{15}=\omega^{2} \sigma_{u} . \\
& A_{21}=c+\frac{\gamma a}{\delta+b-\mu} ; A_{22}=-\sigma_{v}^{2} ; A_{23}=-2 \lambda \sigma_{v} . \\
& A_{31}=-\frac{\omega \sigma_{u}^{2}-\lambda(\rho-1) \sigma_{v}^{2}}{(\delta+b-\mu)^{2}}-\frac{\gamma a}{(\delta+b-\mu)^{2}} ; A_{32}=-\frac{(\rho-1) \sigma_{v}^{2}}{\delta+b-\mu} ; A_{33}=-\frac{2 \lambda(\rho-1) \sigma_{v}}{\delta+b-\mu} ; A_{34}= \\
& \frac{\sigma_{u}^{2}}{\delta+b-\mu} ; A_{35}=\frac{2 \omega \sigma_{u}}{\delta+b-\mu} ; A_{36}=-\frac{\lambda \sigma_{v}}{\delta+b-\mu} . \\
& \quad A_{41}=\left(c+\frac{\gamma a}{\delta+b-\mu}\right)\left(\frac{\lambda a_{y v} \sigma_{v}^{2}-\omega a_{y u} \sigma_{u}^{2}}{\delta}\right) ; A_{42}=a_{p v} \sigma_{v}^{2}\left(b c-\frac{a \gamma(\delta-\mu)}{\delta+b-\mu}\right)-\frac{a_{y v} \sigma_{v}^{2}}{\delta}\left((\mu-b) c+\frac{a \gamma \delta}{\delta+b-\mu}\right) ; \\
& A_{43}=2 a_{y v} a_{p v} \sigma_{v} ; A_{44}=a_{p u} \sigma_{u}^{2}\left(\frac{a \gamma(\delta-\mu)}{\delta+b-\mu}-b c\right)+\frac{a_{y u} \sigma_{u}^{2}}{\delta}\left((\mu-b) c+\frac{a \gamma \delta}{\delta+b-\mu}\right) ; \\
& A_{45}=2 a_{y u} a_{p u} \sigma_{u} ; A_{46}=a_{p v} \sigma_{v}^{2}+\frac{a_{y v}}{\delta} \sigma_{v}^{2} . \\
& \quad A_{52}=a_{y v} \sigma_{v}^{2}\left(b c-\frac{a \gamma(\delta-\mu)}{\delta+b-\mu}\right) ; A_{53}=a_{y v}^{2} \sigma_{v} ; A_{54}=a_{y u} \sigma_{u}^{2}\left(\frac{a \gamma(\delta-\mu)}{\delta+b-\mu}-b c\right) ; A_{55}= \\
& a_{y u}^{2} \sigma_{u} ; A_{56}=a_{y v} \sigma_{v}^{2} . \\
& \quad A_{61}=\left(c+\frac{\gamma a}{\delta+b-\mu}\right)\left(\frac{\lambda a_{p v} \sigma_{v}^{2}-\omega a_{p u} \sigma_{u}^{2}}{\delta}\right) ; A_{62}=-\frac{a_{p v} \sigma_{v}^{2}}{\delta}\left((\mu-b) c+\frac{a \gamma \delta}{\delta+b-\mu}\right) \\
& A_{63}=a_{p v}^{2} \sigma_{v} ; A_{64}=\frac{a_{p u} \sigma_{u}^{2}}{\delta}\left((\mu-b) c+\frac{a \gamma \delta}{\delta+b-\mu}\right) ; A_{65}=a_{p u}^{2} \sigma_{u} ; A_{66}=\frac{a_{p v} \sigma_{v}^{2}}{\delta} . \\
& \quad w_{4}=\frac{\sigma_{u}^{2} a_{y u}}{\delta^{2}}\left(1+(\mu-b) \omega c+\frac{\gamma a \omega \delta}{\delta+b-\mu}\right)+\left(c+\frac{\gamma a}{\delta+b-\mu}\right)\left(\frac{\lambda \sigma_{v}^{2} a_{y v}-\omega \sigma_{u}^{2} a_{y u}}{\delta}\right) \\
& \quad+\frac{\sigma_{v}^{2} a_{y v}}{\delta^{2}}\left(\rho-1-(\mu-b) \lambda c-\frac{\gamma a \lambda \delta}{\delta+b-\mu}\right) ; \\
& \quad w_{6}=\frac{\sigma_{u}^{2} a_{p u}}{\delta^{2}}\left(1+(\mu-b) \omega c+\frac{\gamma a \omega \delta}{\delta+b-\mu}\right)+\left(c+\frac{\gamma a}{\delta+b-\mu}\right)\left(\frac{\lambda \sigma_{v}^{2} a_{p v}-\omega \sigma_{u}^{2} a_{p u}}{\delta}\right) \\
& \quad+\frac{\sigma_{v}^{2} a_{p v}}{\delta^{2}}\left(\rho-1-(\mu-b) \lambda c-\frac{\gamma a \lambda \delta}{\delta+b-\mu}\right) .
\end{aligned}
$$

In the above, $\gamma$ is computed at the correct model: $\gamma=\gamma_{0}$. From there we can compute $q=A^{-1} w$.

To compute the coefficient $m$ in (21) we use (20) and note that

$$
\frac{\partial \gamma}{\partial \varphi}=-\gamma \frac{(\delta+b-\mu)^{2}}{\varphi(\delta+b-\mu)^{2}+a^{2}(\delta-\mu)^{2}}
$$


and that

$$
\begin{aligned}
& \nabla_{v} \gamma=\left(\frac{\partial \gamma}{\partial(\hat{\delta}-\hat{\mu})}, \frac{\partial \gamma}{\partial \hat{\lambda}}, \frac{\partial \gamma}{\partial \hat{\sigma}_{v}}, \frac{\partial \gamma}{\partial \hat{\omega}}, \frac{\partial \gamma}{\partial \hat{\sigma}_{u}}, \frac{\partial \gamma}{\partial \hat{\rho}}\right)^{\prime} \\
& =\frac{a}{\varphi(\delta+b-\mu)^{2}+a^{2}(\delta-\mu)^{2}}\left(\begin{array}{c}
2 \lambda \sigma_{v}^{2} \rho(\delta-\mu)+b \lambda \sigma_{v}^{2}-2(\delta-\mu) \omega \sigma_{u}^{2} \\
\sigma_{v}^{2}\left(\rho(\delta-\mu)^{2}+b(\delta-\mu)\right) \\
2 \sigma_{v} \lambda\left(\rho(\delta-\mu)^{2}+b(\delta-\mu)\right) \\
-(\delta-\mu)^{2} \sigma_{u}^{2} \\
-2(\delta-\mu)^{2} \omega \sigma_{u} \\
(\delta-\mu)^{2} \lambda \sigma_{v}^{2}
\end{array}\right) \\
& -\frac{\gamma}{\varphi(\delta+b-\mu)^{2}+a^{2}(\delta-\mu)^{2}}\left(\begin{array}{c}
\left(2 \varphi(\delta+b-\mu)+2 a^{2}(\delta-\mu)\right) \\
0 \\
0 \\
0 \\
0 \\
0
\end{array}\right) .
\end{aligned}
$$

In particular, the above derivations imply the following expression for SSO:

$$
S S O=\frac{\begin{array}{c}
2 a_{y u}^{2} \sigma_{u}^{2} \sigma_{v}^{2} a_{y v}\left(m q_{6}+m q_{2}\left(b c-a \gamma \frac{\delta-\mu}{\delta+b-\mu}\right)\right)+2 a_{y u}^{2} \sigma_{u}^{2} \sigma_{v} a_{y v}^{2} m q_{3} \\
\left.+2 a_{y u} \sigma_{u}^{2} \sigma_{v}^{2} a_{y v}^{2} m q_{4}\left(b c-a \gamma \frac{\delta-\mu}{\delta+b-\mu}\right)\right)-2 a_{y u}^{2} \sigma_{u} \sigma_{v}^{2} a_{y v}^{2} m q_{5}
\end{array}}{\left(a_{y v}^{2} \sigma_{v}^{2}+a_{y u}^{2} \sigma_{u}^{2}\right)^{2}} .
$$




\section{Figure 1 -- The a/b trade-off}

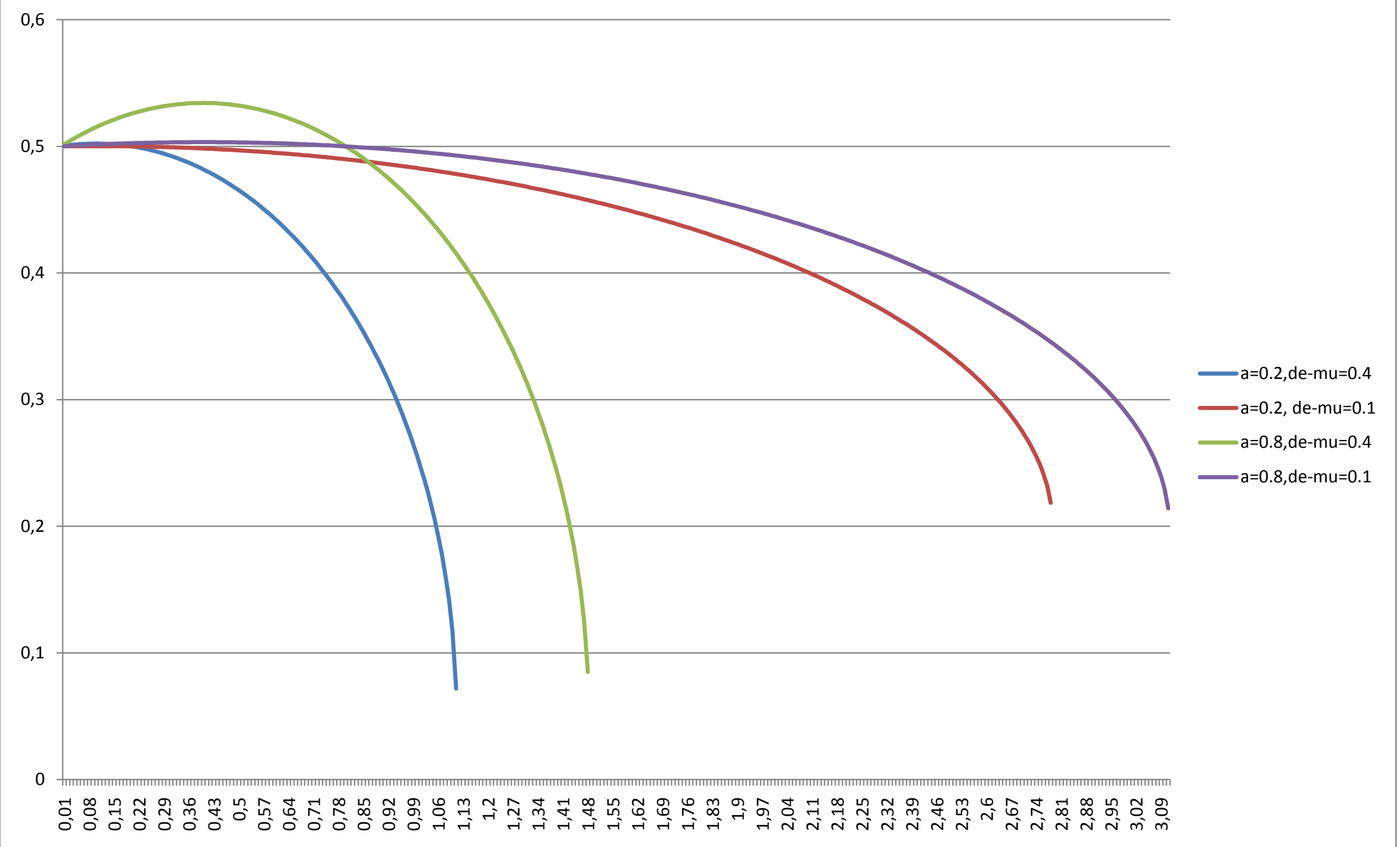




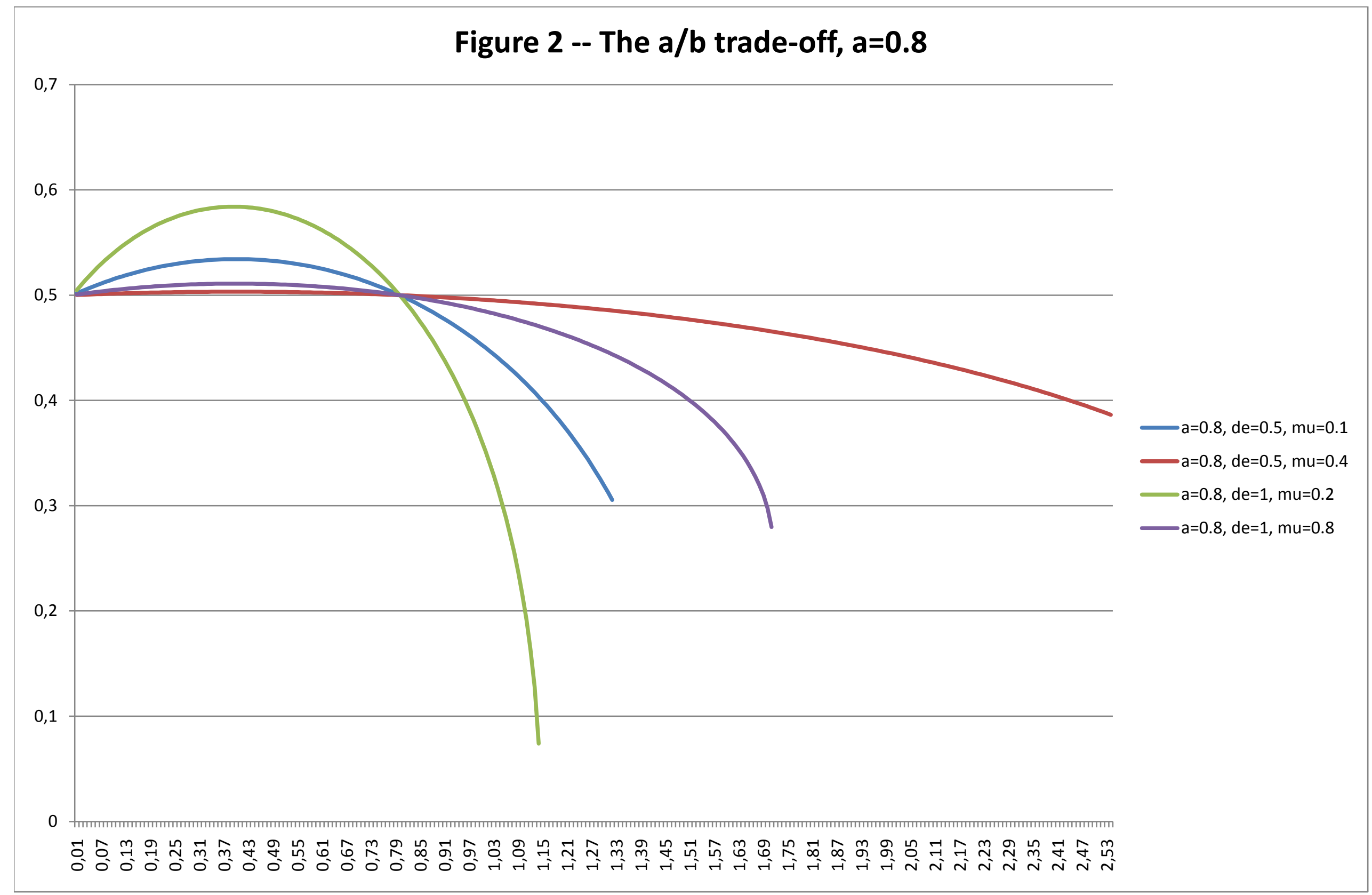


Figure 3 -- Optimal models of left-wing economists, a=0.2

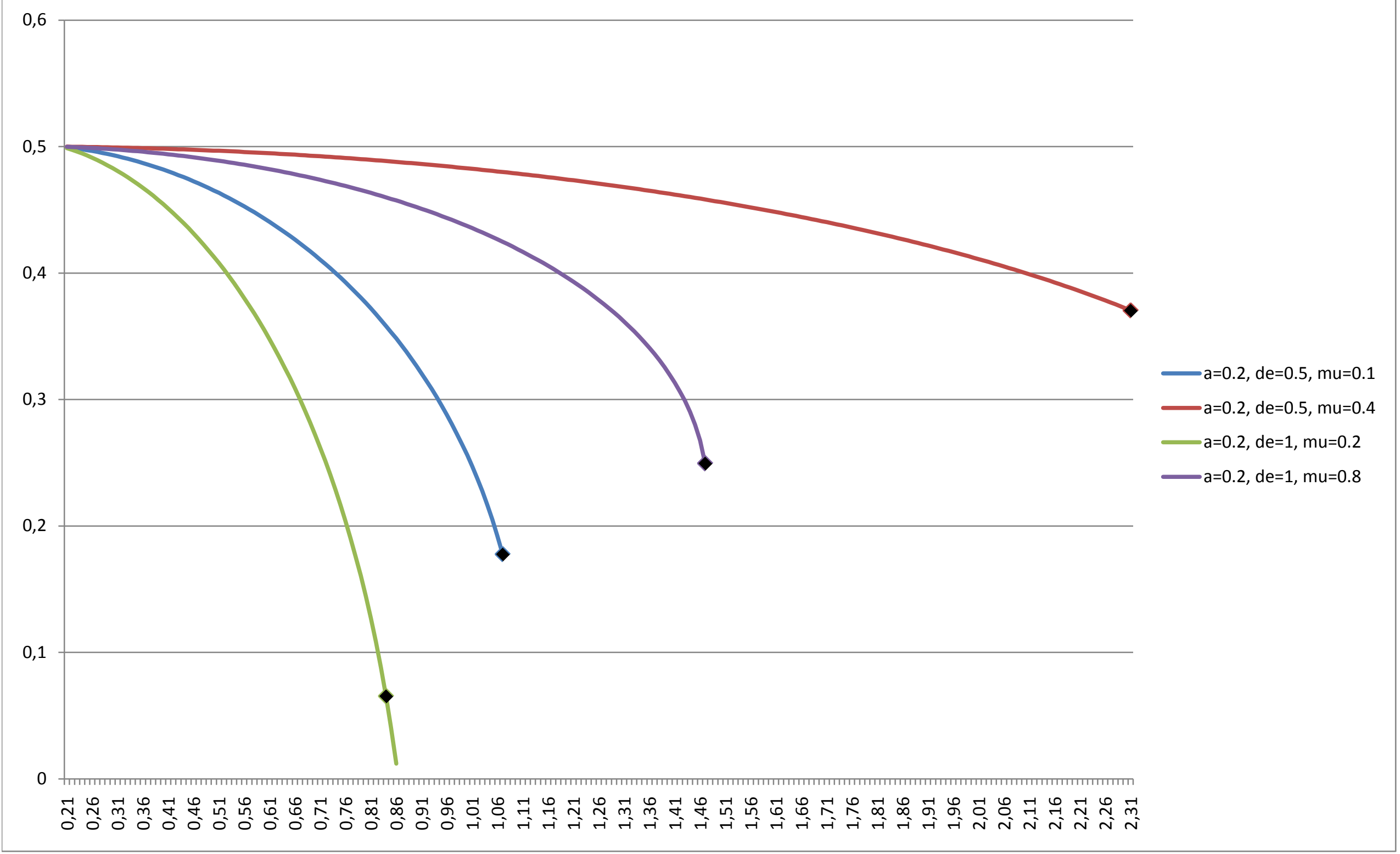


Figure 4 - Ideological sensitivities, $a=0.7 ; b=0.5 ; \omega=1 ; \lambda=1 ; \sigma_{u}{ }^{2}=0.5 ; \delta=$ $0.7 ; \rho=1$.
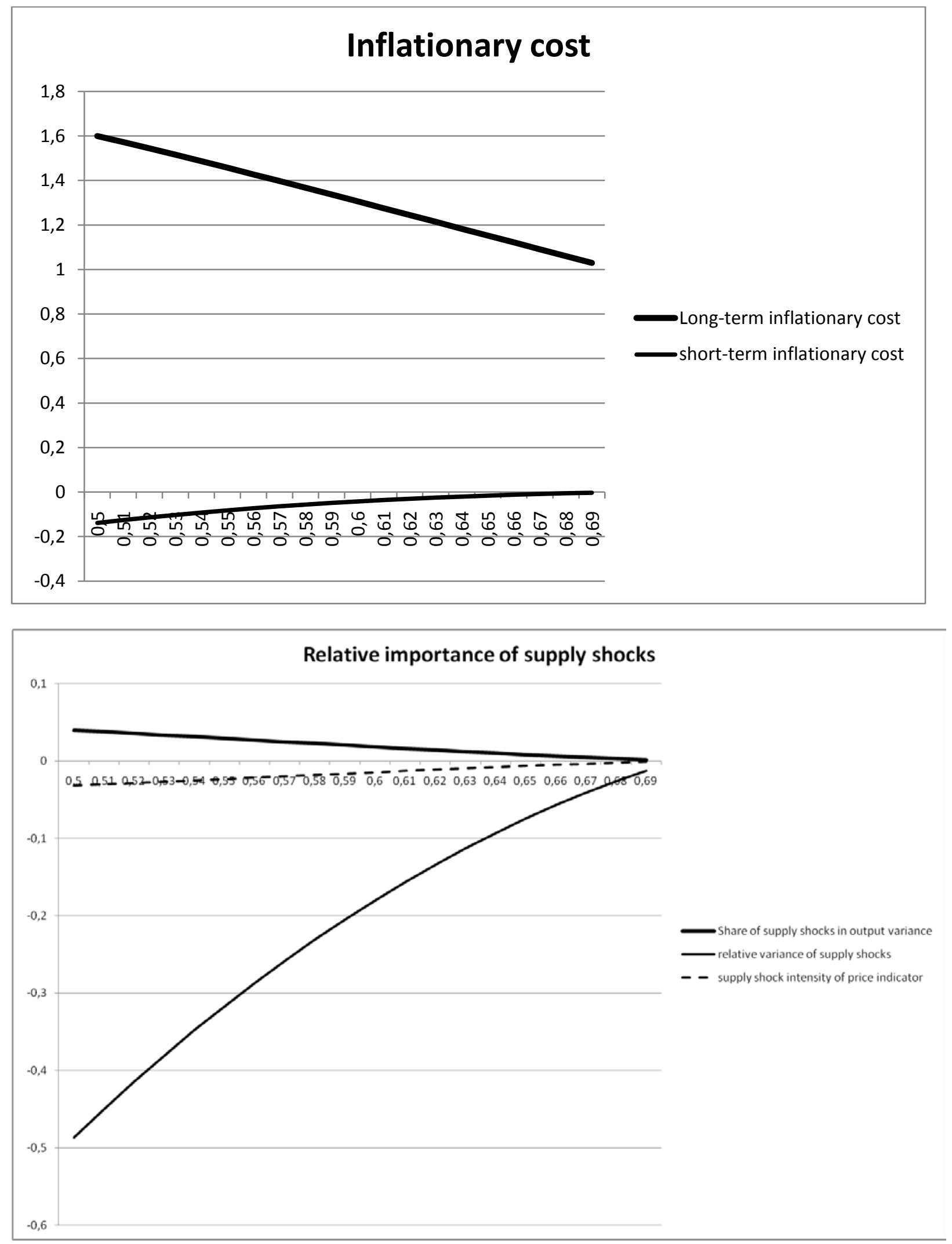
Figure 5 - Ideological sensitivities, $a=0.7 ; b=0.5 ; \omega=1 ; \lambda=1 ; \sigma_{u}{ }^{2}=0.9 ; \delta=$ $0.7 ; \rho=1$.
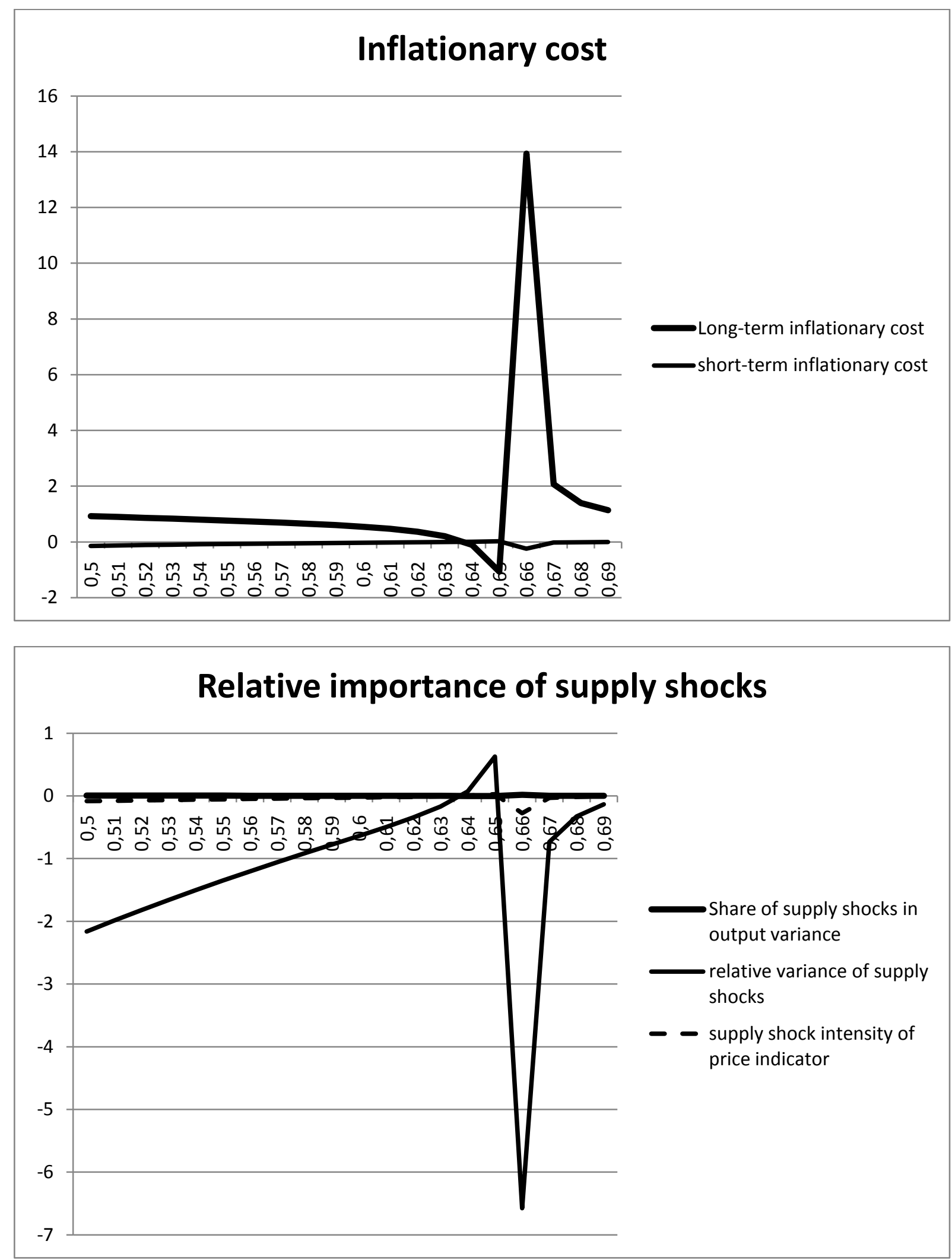
Figure 6 - Ideological sensitivities, $a=0.7 ; b=0.5 ; \omega=1 ; \lambda=1 ; \sigma_{u}{ }^{2}=0.1 ; \delta=$ $0.7 ; \rho=1$.
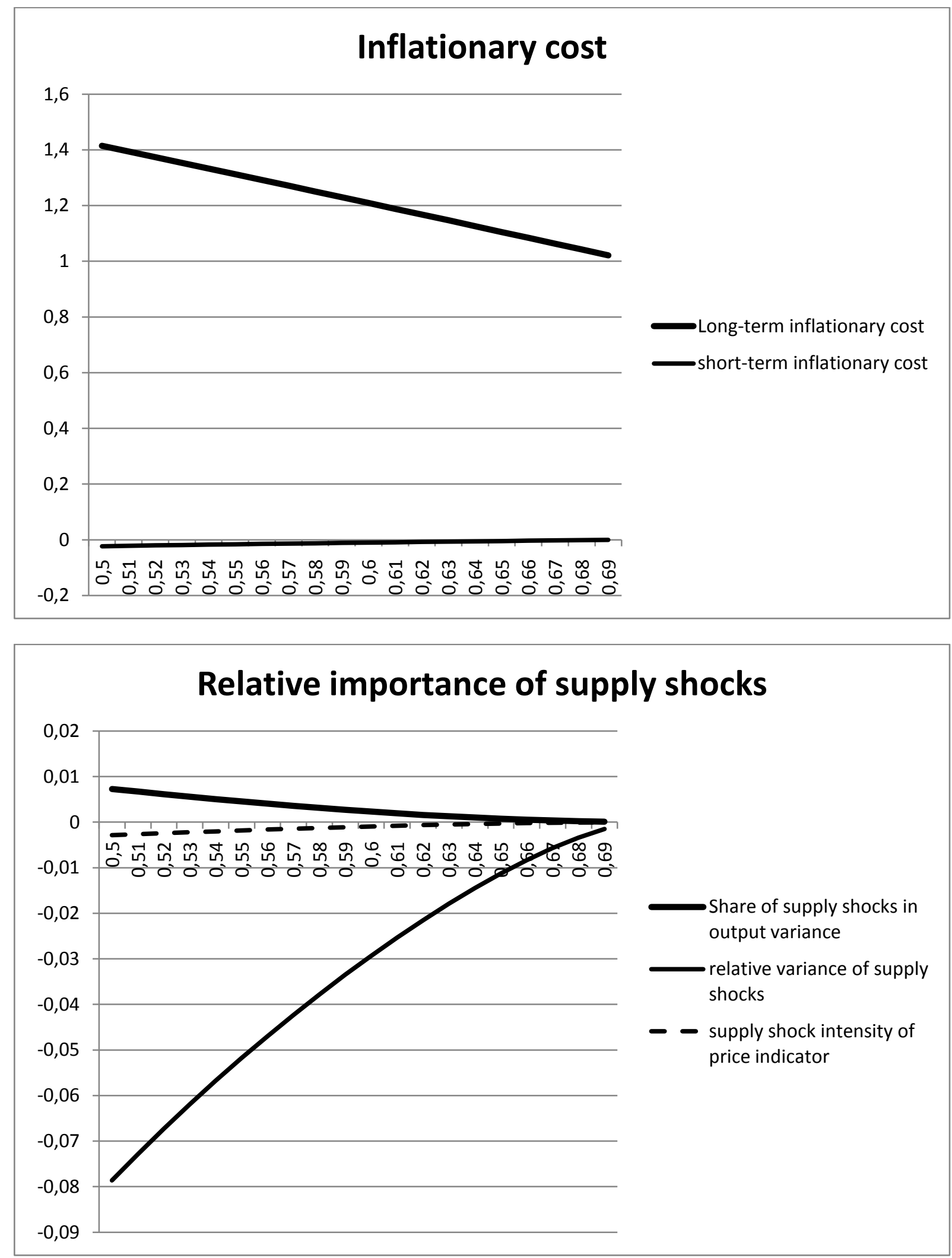
Figure 7-Ideological sensitivities, $a=1 ; b=0.5 ; \omega=1 ; \lambda=1 ; \sigma_{u}{ }^{2}=0.5 ; \delta=$ $0.7 ; \rho=1$.
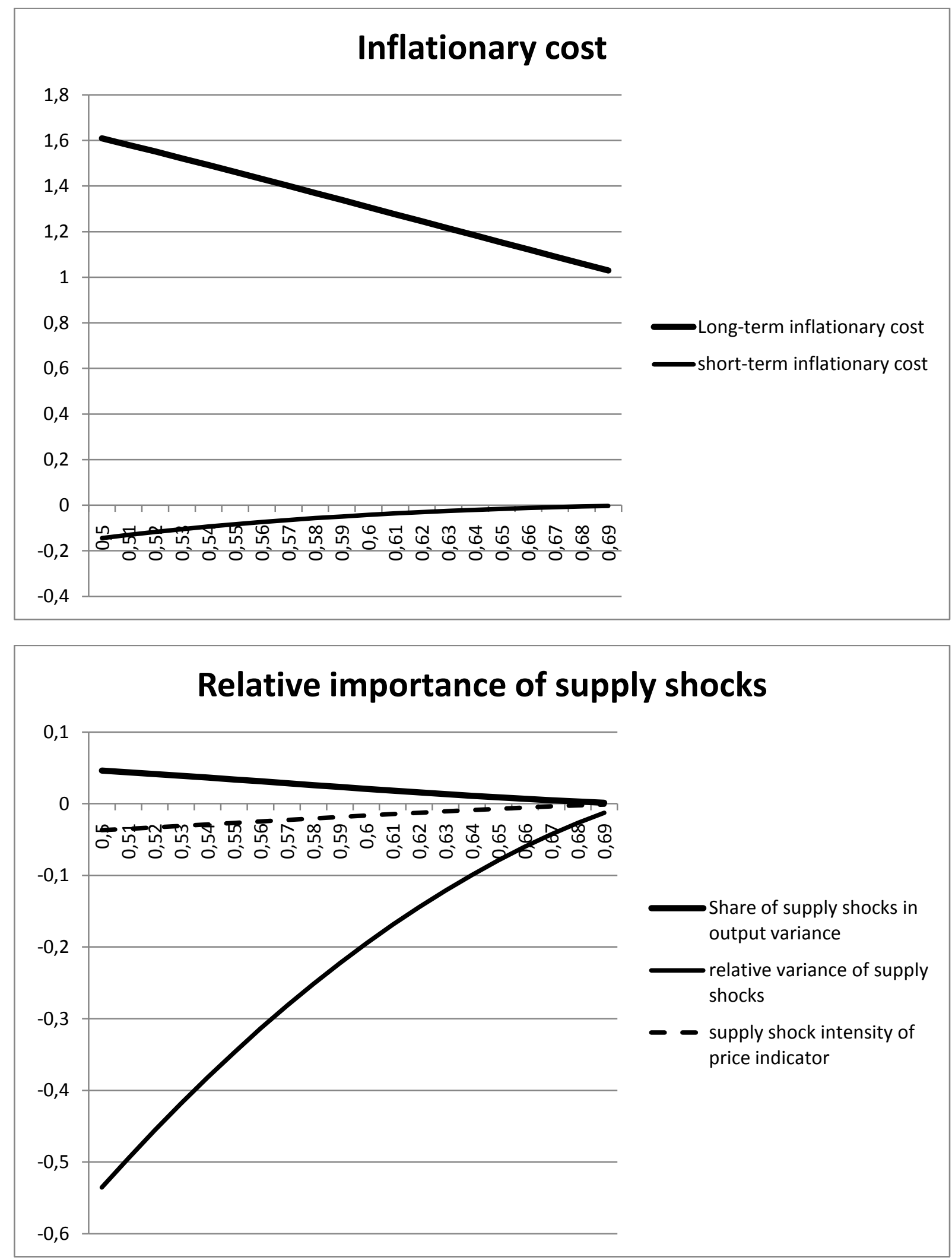
Figure 8 - Ideological sensitivities, $a=0.3 ; b=0.5 ; \omega=1 ; \lambda=1 ; \sigma_{u}{ }^{2}=0.5 ; \delta=$ $0.7 ; \rho=1$.
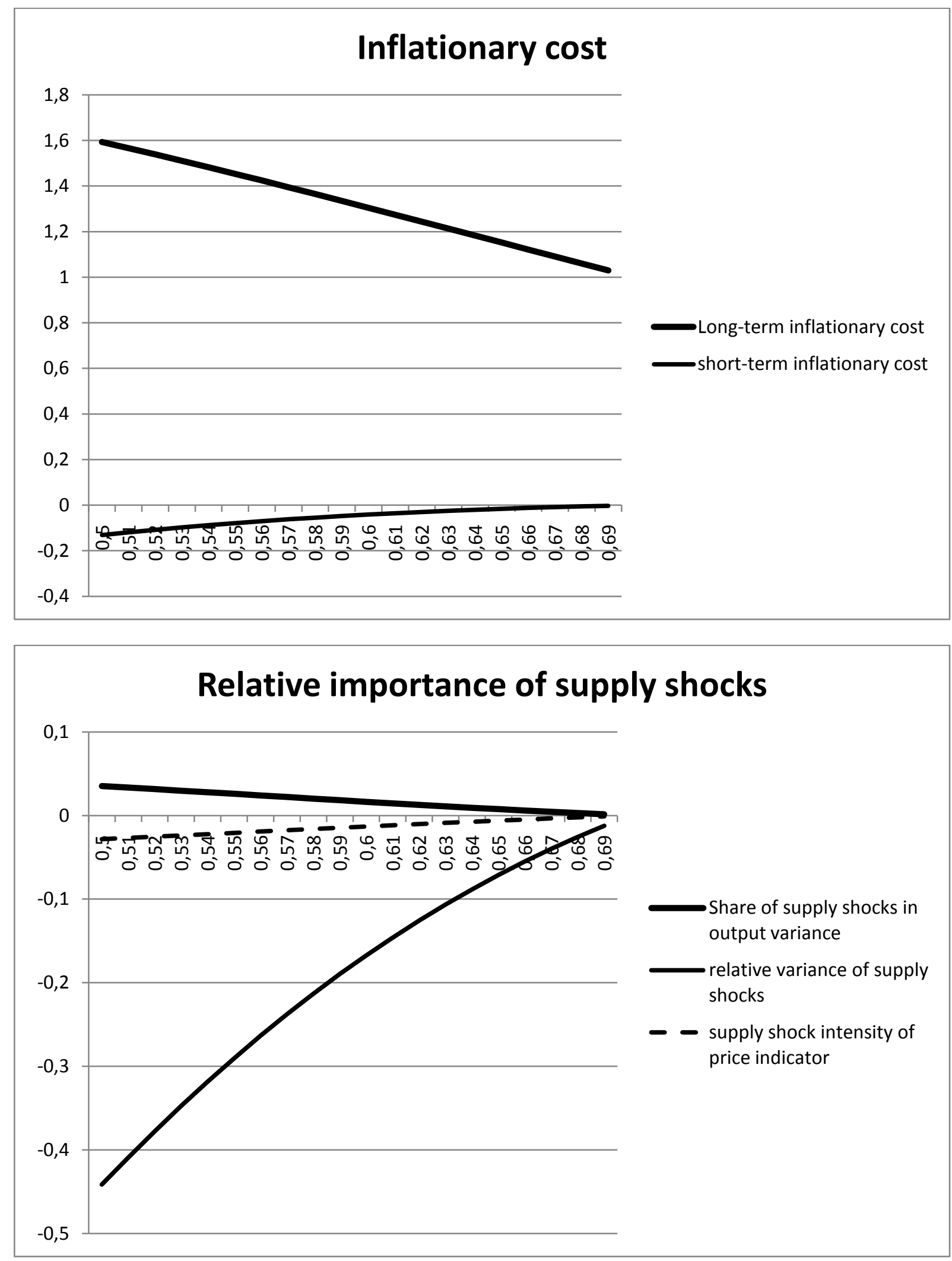
Figure 9 - Ideological sensitivities, $a=0.7 ; b=0.1 ; \omega=1 ; \lambda=1 ; \sigma_{u}{ }^{2}=0.5 ; \delta=$ $0.7 ; \rho=1$.
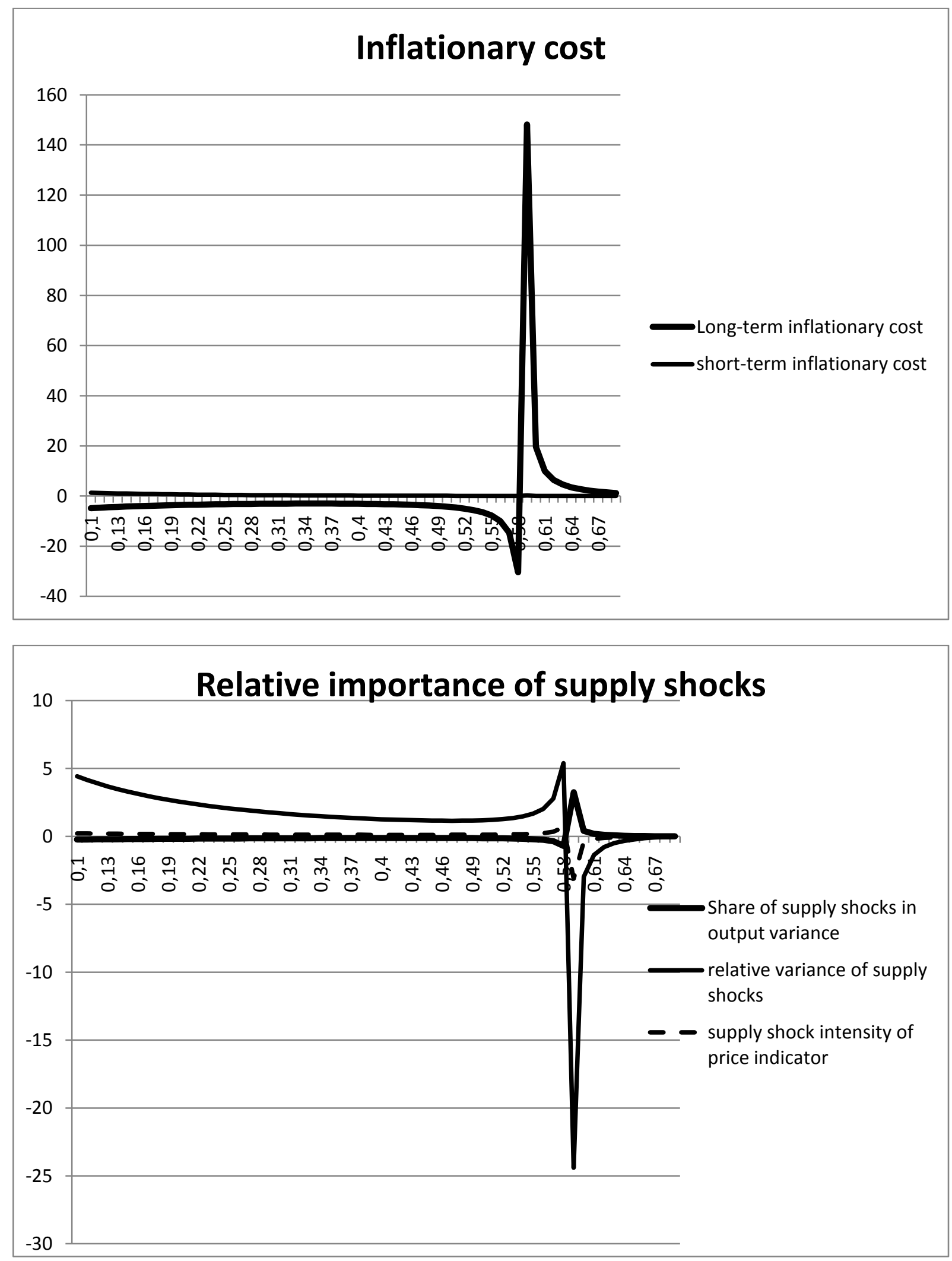